\title{
$\phi$ and $\Omega$ production in relativistic heavy-ion collisions in a dynamical quark coalescence model
}

\author{
Lie-Wen Chen ${ }^{1,2}$ and Che Ming $\mathrm{Ko}^{3}$ \\ ${ }^{1}$ Institute of Theoretical Physics, Shanghai Jiao Tong University, Shanghai 200240, China \\ ${ }^{2}$ Center of Theoretical Nuclear Physics, National Laboratory of Heavy Ion Accelerator, Lanzhou 730000, China \\ ${ }^{3}$ Cyclotron Institute and Physics Department, Texas A\&M University, College Station, Texas 77843-3366, USA
}

(Received 9 February 2006; published 17 April 2006)

\begin{abstract}
Based on the phase-space information obtained from a multiphase transport model within the string-melting scenario for strange and antistrange quarks, we study the yields and transverse-momentum spectra of $\phi$ mesons and $\Omega\left(\Omega^{-}+\bar{\Omega}^{+}\right)$baryons and their anisotropic flows in $\mathrm{Au}+\mathrm{Au}$ collisions at RHIC using a dynamical quark coalescence model that includes the effect from quark phase-space distributions inside hadrons. With current quark masses and fixing the $\phi$ and $\Omega$ radii from fitting measured yields, we first study the ratio of the yield of $\Omega$ baryons to that of $\phi$ mesons as well as their elliptic and fourth-order flows as functions of their transverse momentum. How the elliptic and fourth-order flows of $\phi$ mesons and $\Omega$ baryons are related to those of strange and antistrange quarks is then examined. The dependence of these results on $\phi$ and $\Omega$ radii as well as on the strange quark mass is also studied.
\end{abstract}

DOI: 10.1103/PhysRevC.73.044903

PACS number(s): 12.38.Mh, 24.10.Lx, 25.75.Ld

\section{INTRODUCTION}

Recently, there has been a lot of interest in using the quark coalescence or recombination model to understand the experimental data from heavy-ion collisions at the Relativistic Heavy Ion Collider (RHIC). As shown in Refs. [1-4], the quark coalescence model can explain successfully the observed anomalously large enhancement of baryon to meson ratio at intermediate transverse momenta and scaling of the elliptic flow of identified hadrons according to their valence quark numbers. Most of these applications of the quark coalescence model were based on a simple momentum-space coalescence in which only quarks with the same momentum can coalesce into hadrons. Also, the phase-space information of quarks in the partonic matter produced in relativistic heavy-ion collisions is usually taken from a schematic fireball model. Although the fireball model was still used in Ref. [1], the effect from quark momentum spread and spatial distribution inside hadrons was included in calculating the probabilities for quarks to coalesce to hadrons. Only in Ref. [5] was the coalescence probability calculated also with quark phase-space information from a dynamical parton cascade model. Results from such a dynamical quark coalescence model indicate that the phasespace structure of quarks at freeze-out plays an important role in hadron production from the quark-gluon plasma (QGP). These studies were mainly concerned with hadrons that consist of light quarks and/or heavy charm quarks. In the present study, we use the dynamical quark coalescence model, which is based on the quark phase-space information from a multiphase transport (AMPT) model within the string-melting scenario and includes the quark structure of hadrons, to study instead the production and anisotropic flow of $\phi$ mesons and $\Omega\left(\Omega^{-}+\bar{\Omega}^{+}\right)$baryons that consist of strange quarks in $\mathrm{Au}+\mathrm{Au}$ collisions at RHIC.

The study of $\phi$ meson and $\Omega$ baryon production in relativistic heavy-ion collisions is a topic of great interest since one of the signatures for the QGP produced in relativistic heavy-ion collisions is enhanced production of hadrons consisting of strange and/or antistrange quarks. The enhancement occurs because masses of strange quarks are comparable to the temperature of the QGP and are thus expected to be abundantly produced from quark and gluon inelastic scattering once the QGP is formed in the collisions [6,7]. Since the $\phi$ meson carries hidden strangeness $(s \bar{s})$ and the $\Omega$ baryon consists of three valence strange quarks (sss) or antistrange quarks $(\bar{s} \bar{s} \bar{s})$, their production in heavy-ion collisions would also be enhanced if the QGP is formed.

Furthermore, anisotropic flows of $\phi$ mesons and $\Omega$ baryons in relativistic heavy-ion collisions are useful for understanding the collective dynamics of strange quarks in the produced QGP [8,9]. It is known that anisotropic flows are sensitive to the properties of dense matter formed during the early stage of heavy-ion collisions [10-13]. This sensitivity exists not only in the elliptic flow $v_{2}$ [14-21] but also in the smaller higher order anisotropic flows, such as the fourth-order anisotropic flow $v_{4}$ [22-29]. Moreover, observed hadron elliptic flows in heavy-ion collisions at RHIC were found to satisfy the valence quark number scaling; that is, the elliptic flow per quark is the same at the same transverse momentum per quark. As shown in Refs. $[1,3,4]$, such a scaling of hadron elliptic flows according to their valence quark numbers can be understood in the quark recombination/coalescence model. Other scaling relations among hadron anisotropic flows, such as $v_{4}\left(p_{T}\right) \sim$ $v_{2}^{2}\left(p_{T}\right)$, have also been observed in experimental data [26,27], and they have been shown in the quark coalescence model to relate to similar scaling relations among quark anisotropic flows [28,29]. Because of their small scattering cross sections with other hadrons [30-36], $\phi$ mesons and $\Omega$ baryons are little affected by rescattering effects in later hadronic stage of the collision and are thus expected to provide more direct information on the properties of the QGP and how they are produced during hadronization [37-40].

Our study shows that in heavy-ion collisions at RHIC the ratio of the yield of $\Omega$ baryons to that of $\phi$ mesons at 
intermediate transverse momenta is strongly enhanced relative to that at low transverse momenta, similar to that observed in the data for the proton to pion ratio. Also, the elliptic flows $v_{2}\left(p_{T}\right)$ of $\phi$ mesons and $\Omega$ baryons follow approximately the valence quark number scaling. Their valence quark number scaled elliptic flows deviate, however, strongly from the elliptic flow of strange and antistrange quarks. For the fourth-order anisotropic flow $v_{4}\left(p_{T}\right)$, it scales with the square of the elliptic flow for both $\phi$ mesons and $\Omega$ baryons. We have also studied the dependence of these results on the radii of $\phi$ meson and $\Omega$ baryon as well as on the strange quark mass. For physically reasonable radii, yields of both $\phi$ mesons and $\Omega$ baryons increase with increasing radii. Their yields eventually decrease as the radii become unrealistically large. The scaled elliptic flows of both $\phi$ mesons and $\Omega$ baryons increase with increasing radii and approach that of strange and antistrange quarks for very large radii as in the naive momentum-space quark coalescence model. Changing strange and antistrange quark masses to their constituent quark masses affects significantly the yields of $\phi$ mesons and $\Omega$ baryons but has little effect on their anisotropic flows. Our results therefore suggest that both the dynamical phase-space information of partons at freeze-out and quark structure of hadrons are important for $\phi$ meson and $\Omega$ baryon production in relativistic heavy-ion collisions. We note that our study based on the dynamical quark coalescence model for $\phi$ meson production and elliptic flow in relativistic heavy-ion collisions differs from that of Ref. [8], where $\phi$ mesons were reconstructed from the invariant mass distributions of kaons and antikaons from the AMPT model.

The paper is organized as follows. In Sec. II, we briefly review the AMPT model and present detailed information on the dynamics of quarks and antiquarks (also called partons) in heavy-ion collisions at RHIC. In Sec. III, the dynamical quark coalescence model based on parton phase-space distributions at freeze-out is described for $\phi$ meson and $\Omega$ baryon production. This also includes the construction of the quark Wigner phase-space functions inside the $\phi$ meson and the $\Omega$ baryon. The dynamical quark coalescence model is then used in Sec. IV to study the yields and transverse-momentum spectra as well as the anisotropic flows of $\phi$ mesons and $\Omega$ baryons in $\mathrm{Au}+$ $\mathrm{Au}$ collisions at RHIC. In Sec. V, the dependence of these results on the radii of the $\phi$ meson and $\Omega$ baryon is studied. The effect of change in the strange quark mass is studied in Sec. VI. Finally, we conclude with a summary in Sec. VII.

\section{PARTONIC DYNAMICS IN HEAVY-ION COLLISIONS AT RHIC}

The dynamical quark coalescence model used in the present study requires the space-time and momentum information of quarks and antiquarks during hadronization of the produced QGP in relativistic heavy-ion collisions. In particular, the dynamics of strange and antistrange quarks are needed for describing the production of $\phi$ mesons and $\Omega$ baryons as well as their anisotropic flows, and they are taken from the AMPT model within the string-melting scenario [41].

\section{A. The AMPT model}

The AMPT model is a hybrid model that uses minijet partons from hard processes and strings from soft processes in the Heavy Ion Jet Interaction Generator (HIJING) model [42] as the initial conditions for modeling heavy-ion collisions at ultrarelativistic energies. The time evolution of resulting minijet partons, which are mostly gluons, is described by Zhang's parton cascade (ZPC) [43] model. At present, this model includes only parton-parton elastic scatterings with in-medium cross sections derived from the lowest order Born diagrams and having magnitude and angular distribution fixed by treating the gluon screening mass as a parameter. After minijet partons stop interacting, they are combined with their parent strings, as in the HIJING model with jet quenching, to fragment into hadrons using the Lund string fragmentation model as implemented in the PYTHIA program [44]. The final-state hadronic scatterings are modeled by a relativistic transport (ART) model [45].

Since the initial energy density in heavy-ion collisions at RHIC is much larger than the critical energy density at which the hadronic matter to QGP transition would occur [46-48], the AMPT model has been extended by converting initial excited strings into partons [18]. In this string-melting scenario, hadrons that would have been produced from string fragmentation are converted instead to their valence quarks and/or antiquarks. Interactions among these quarks are again described by the ZPC parton cascade model. Since inelastic scatterings are at present not included, the resulting partonic matter consists of only quarks and antiquarks from melted strings. To take into account the effect of stronger scattering among gluons if they were present, the scattering cross sections between quarks and antiquarks are taken to be the same as those for gluons. These quarks and antiquarks are converted to hadrons when they stop scattering with other partons. For a parton cross section of $10 \mathrm{mb}$, which is used in the present study, this criterion for hadronization is not too different from the one based on the condition that the local energy density of hadronizing partons is at the critical energy density given by lattice QCD calculations. The transition from partonic matter to hadronic matter is achieved using a simple coalescence model, which combines the two nearest quark and antiquark into mesons and three nearest quarks or antiquarks into baryons or antibaryons that are close to the invariant mass of these partons. Details of the AMPT model can be found in Ref. [41].

In the present study, we use the AMPT model in the string-melting scenario with the default current quark mass of $9.9 \mathrm{MeV}$ for the $d$ quark, $5.6 \mathrm{MeV}$ for the $u$ quark, and $199 \mathrm{MeV}$ for the $s$ quark and a constant parton scattering cross section of $10 \mathrm{mb}$. We note that using parton scattering cross sections of 6-10 mb, the AMPT model with string melting was able to reproduce both the centrality and transversemomentum (below $2 \mathrm{GeV} / c$ ) dependence of the elliptic flow [18] and pion interferometry [49] measured in $\mathrm{Au}+\mathrm{Au}$ collisions at $\sqrt{s_{N N}}=130 \mathrm{GeV}$ at RHIC $[50,51]$ as well as the measured $p_{T}$ dependence of both $v_{2}$ and $v_{4}$ of midrapidity charged hadrons in the same collision at $\sqrt{s_{N N}}=200 \mathrm{GeV}$ [28]. 




FIG. 1. (Color online) Rapidity distributions of strange and antistrange quarks $(s+\bar{s})$ as well as light quarks and antiquarks $(u+\bar{u}+d+\bar{d})$ at freeze-out in $\mathrm{Au}+\mathrm{Au}$ collisions at $\sqrt{s_{N N}}=$ $200 \mathrm{GeV}$ and $b=8 \mathrm{fm}$.

\section{B. Spectrum of strange and antistrange quarks}

We first show in Fig. 1 the rapidity distributions of strange quarks and antiquarks $(s+\bar{s})$ as well as light quarks and antiquarks $(u+\bar{u}+d+\bar{d})$ at freeze-out (i.e., the point when scattering with other partons stops) in $\mathrm{Au}+\mathrm{Au}$ collisions at $\sqrt{s_{N N}}=200 \mathrm{GeV}$ and $b=8 \mathrm{fm}$. It is seen that the rapidity density $d N / d y$ at midrapidity is about 94 for strange quarks and antiquarks and about 770 for light quarks and antiquarks. Since inelastic partonic scatterings are absent in the present model, all these quarks and antiquarks are from melted strings during the initial stage of the collisions.

The transverse-momentum distributions of midrapidity strange and antistrange quarks as well as light quarks and antiquarks at freeze-out in the same collision are shown in Fig. 2. The figure clearly shows a quark mass effect as strange



FIG. 2. (Color online) Transverse-momentum distributions of midrapidity strange and antistrange quarks $(s+\bar{s})$ as well as light quarks and antiquarks $(u+\bar{u}+d+\bar{d})$ at freeze-out in $\mathrm{Au}+\mathrm{Au}$ collisions at $\sqrt{s_{N N}}=200 \mathrm{GeV}$ and $b=8 \mathrm{fm}$. and antistrange quarks display a stiffer transverse-momentum distribution at low $p_{T}$ compared to that of light quarks and antiquarks. Fitting the transverse-momentum spectrum by a Boltzmann distribution, we find that the effective temperature of the partonic matter at freeze-out is about $161 \mathrm{MeV}$, which is consistent with the predicted critical temperature $T_{c} \approx 150-180 \mathrm{MeV}$ from the lattice QCD for the QGP to hadronic matter phase transition [52,53].

\section{Space-time structure of strange and antistrange quarks at freeze-out}

The spatial distribution of midrapidity strange and antistrange quarks at freeze-out in this collision is shown in Fig. 3, with the upper panel displaying their number density distribution in the transverse plane and the lower panel giving the corresponding contour plot. Here, the transverse plain refers to the $x-y$ plane with the $x$ axis pointing in the direction of the impact parameter and the $y$ axis perpendicular to the $x$ axis as well as the beam direction. It is seen that the number density of strange and antistrange quarks at freeze-out peaks approximately at a circle with a radius of about $4 \mathrm{fm}$ in the transverse plane, implying that these partons mainly freeze out from the surface of an expanding fireball where the number density is roughly between 1.2 and $1.4 \mathrm{fm}^{-2}$ in the transverse plane.
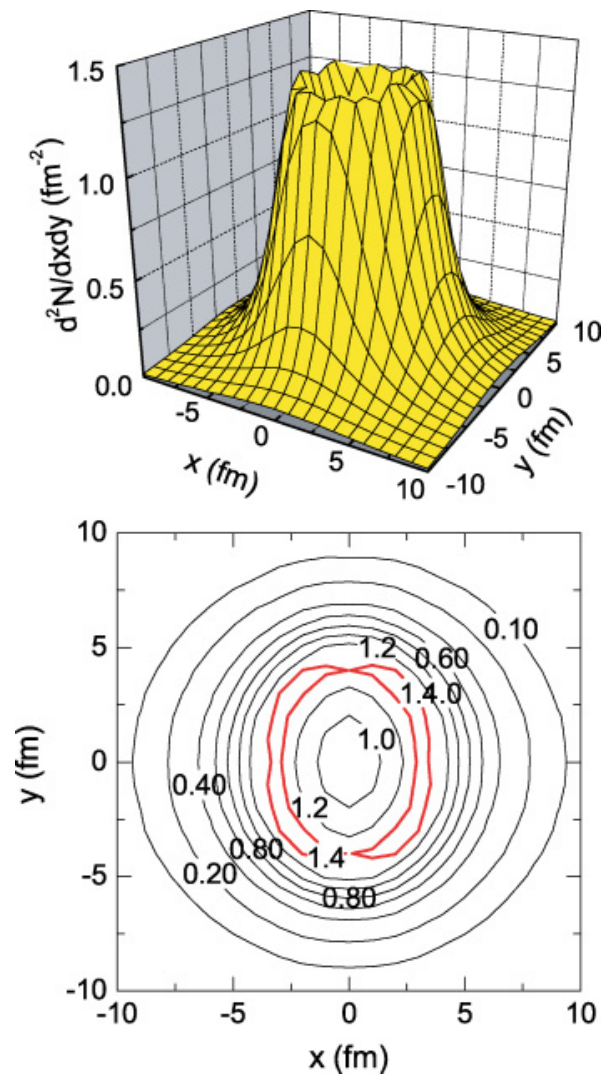

FIG. 3. (Color online) Number density distribution in the transverse plane for midrapidity strange and antistrange quarks at freezeout in $\mathrm{Au}+\mathrm{Au}$ collisions at $\sqrt{s_{N N}}=200 \mathrm{GeV}$ and $b=8 \mathrm{fm}$ (upper panel) and corresponding contour plot (lower panel). 


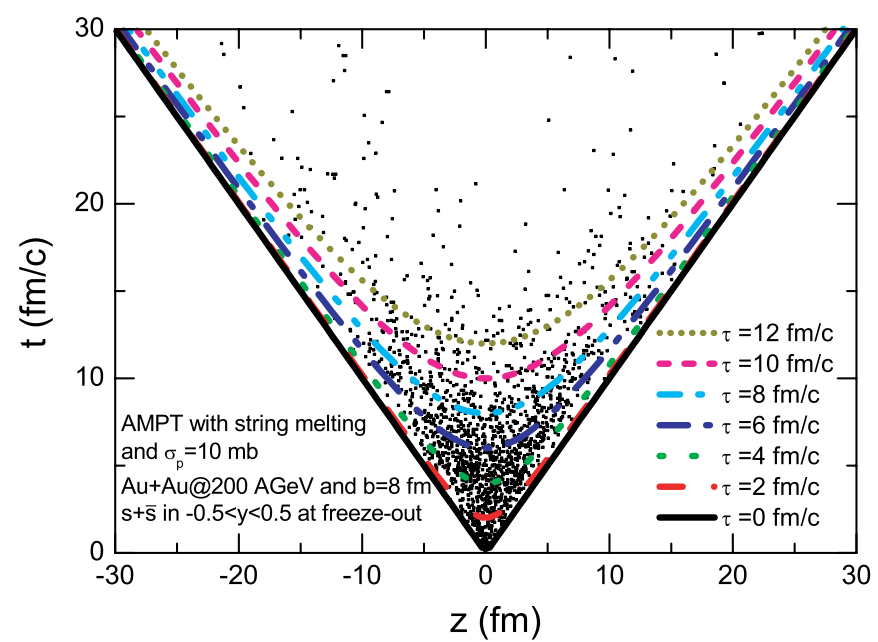

FIG. 4. (Color online) Space-time structure of midrapidity strange and antistrange quarks at freeze-out in $\mathrm{Au}+\mathrm{Au}$ collisions at $\sqrt{s_{N N}}=200 \mathrm{GeV}$ and $b=8 \mathrm{fm}$.

The space-time structure of $z$ - $t$ correlations for midrapidity strange and antistrange quarks at freeze-out in this collision is shown in Fig. 4. Instead of a sudden freeze-out, these partons are seen to freeze out continuously in the proper time $\tau$, with most between $\tau=4$ and $6 \mathrm{fm} / c$. This can be more clearly seen from their freeze-out rate as a function of proper time $\tau$ or the time $t$ in the nucleus-nucleus center-of-mass system as shown in Fig. 5. It shows that the freeze-out rate of midrapidity strange and antistrange quarks peaks at $\tau=5 \mathrm{fm} / c$ or $t=5.5 \mathrm{fm} / c$.

The AMPT model also provides information on the time evolution of parton energy density. This is shown in Fig. 6 for the central cell of the partonic matter in the collision discussed. As in Refs. [46,47], the central cell is taken to have a transverse radius of $1 \mathrm{fm}$ and a longitudinal dimension of $5 \%$ of the time $t$ after the two nuclei have fully overlapped in the longitudinal direction. It is seen that the central energy density is about



FIG. 5. (Color online) Freeze-out rate of midrapidity strange and antistrange quarks as a function of proper time $\tau$ as well as the time $t$ in the nucleus-nucleus center-of-mass system.

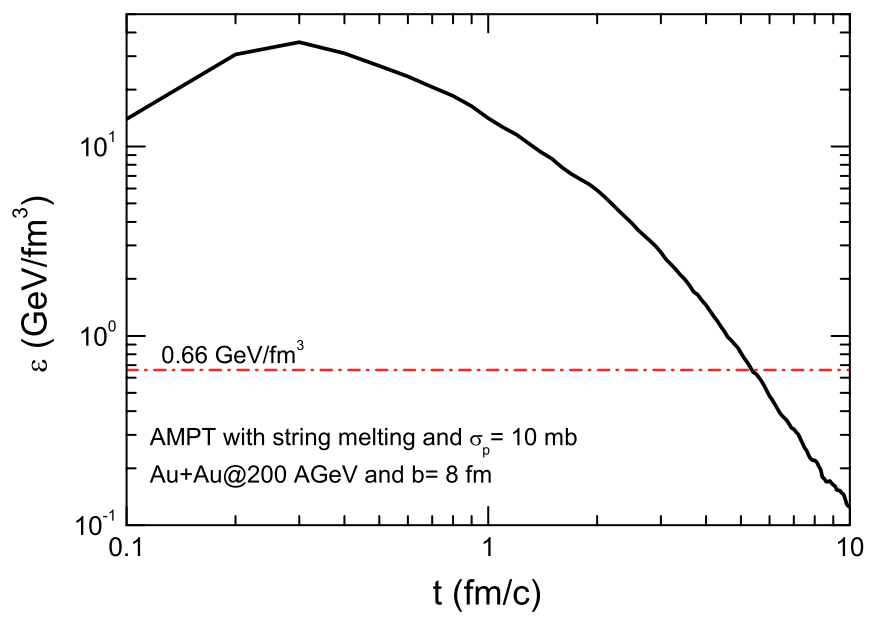

FIG. 6. (Color online) Time evolution of the energy density in the central cell of partonic matter in $\mathrm{Au}+\mathrm{Au}$ collisions at $\sqrt{s_{N N}}=$ $200 \mathrm{GeV}$ and $b=8 \mathrm{fm}$.

$0.6 \mathrm{GeV} / \mathrm{fm}^{3}$ at $t=5.5 \mathrm{fm} / c$ when most of partons freeze out. This energy density is consistent with the critical energy density of about $0.66 \mathrm{GeV} / \mathrm{fm}^{3}$ predicted by the lattice QCD for the QGP to hadronic matter transition $[52,53]$.

\section{Anisotropic flow of strange and antistrange quarks}

Anisotropy in the transverse-momentum distribution of particles in noncentral heavy-ion collisions is generated by the pressure anisotropy in the initial compressed matter [54,55] and thus depends on the geometry and energy density as well as the properties of produced matter during the early stage of these collisions. For $\phi$ mesons and $\Omega$ baryons, their anisotropic flows are essentially determined by those of strange and antistrange quarks at freeze-out and their hadronization mechanism. As discussed previously, the effect of later hadronic scattering is small because the interactions of $\phi$ mesons and $\Omega$ baryons in the hadronic matter are relatively weak and also the pressure becomes more isotropic during the hadronic stage of the collisions.

From the momentum distribution of strange and antistrange quarks at freeze-out, their elliptic and fourth-order anisotropic flows can be evaluated according to the following singleparticle averages:

$$
\begin{aligned}
& v_{2}\left(p_{T}\right)=\left\langle\frac{p_{x}^{2}-p_{y}^{2}}{p_{T}^{2}}\right\rangle, \\
& v_{4}\left(p_{T}\right)=\left\langle\frac{p_{x}^{4}-6 p_{x}^{2} p_{y}^{2}+p_{y}^{4}}{p_{T}^{4}}\right\rangle,
\end{aligned}
$$

where $p_{x}$ and $p_{y}$ are, respectively, projections of their momentum in and perpendicular to the reaction plane, defined by the beam and impact parameter axes, and $p_{T}=\left(p_{x}^{2}+p_{y}^{2}\right)^{1 / 2}$ is the transverse momentum.

In Fig. 7, we show the $p_{T}$ dependence of $v_{2}$ and $v_{4}$ of midrapidity strange and antistrange quarks $(s+\bar{s})$ at freezeout in $\mathrm{Au}+\mathrm{Au}$ collisions at $\sqrt{s_{N N}}=200 \mathrm{GeV}$ and $b=8 \mathrm{fm}$. It is seen that strange and antistrange quarks exhibit not only a 


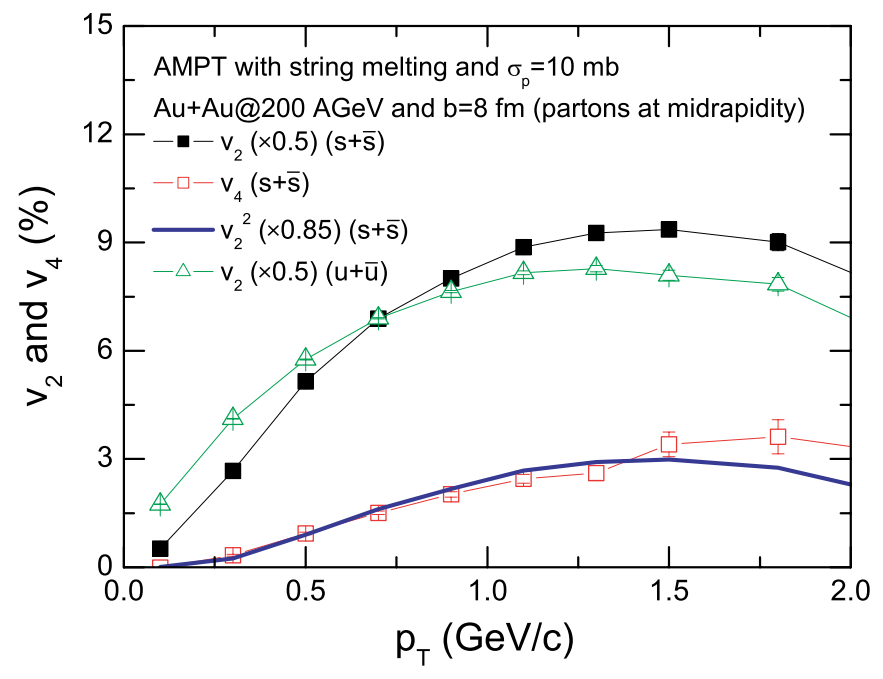

FIG. 7. (Color online) Transverse-momentum dependence of elliptic flow $v_{2}$ (solid squares) and forth-order anisotropic flow $v_{4}$ (open squares) of midrapidity strange and antistrange quarks $(s+\bar{s})$ at freeze-out in $\mathrm{Au}+\mathrm{Au}$ collisions at $\sqrt{s_{N N}}=200 \mathrm{GeV}$ and $b=8 \mathrm{fm}$. The thick solid line represents $0.85 v_{2}^{2}$ of strange and antistrange quarks, and open triangles are $v_{2}$ of midrapidity light up and antiup quarks $(u+\bar{u})$.

strong $v_{2}$ but also a non-negligible $v_{4}$ that approximately scales with $v_{2}^{2}$ with a scaling coefficient of 0.85 (i.e, $v_{4} \sim 0.85 v_{2}^{2}$ as shown by the thick solid line in Fig. 7). Compared to the $v_{2}$ of midrapidity light up and antiup quarks $(u+\bar{u})$, shown by triangles in Fig. 7 , the $v_{2}$ of heavier strange and antistrange quarks has a smaller value at low $p_{T}$ but a larger value at high $p_{T}$, similar to the mass ordering of hadron elliptic flows in the hydrodynamic model in which the produced matter is assumed to be in local thermal equilibrium and thus develops a large collective radial flow. However, instead of a continuing increase of $v_{2}$ with respect to $p_{T}$ as in the hydrodynamic model, the $v_{2}$ of partons in the transport model saturates at a maximum value when their $p_{T}$ becomes large, indicating that high-momentum partons do not reach thermal equilibrium with the bulk of the partonic matter.

\section{A DYNAMICAL QUARK COALESCENCE MODEL FOR $\phi$ AND $\Omega$ PRODUCTION}

In the coalescence model, the probability for forming a bound cluster from a many-particle system is determined by the overlap of the wave functions of coalescing particles with the internal wave function of the cluster. Its validity is based on the assumption that coalescing particles are statistically independent and the binding energy of formed cluster and the quantum dynamics of the coalescing process play only minor roles [56]. In the present study, we assume that correlations among partons at freeze-out are weak and binding energies of formed hadrons can be neglected. Furthermore, the coalescence model is considered as a perturbative approach, valid only if the number of partons coalesced into hadrons is small compared with the total number of partons in the system. This condition is satisfied for $\phi$ mesons and $\Omega$ baryons produced in relativistic heavy-ion collisions as their numbers measured in experiments are indeed significantly smaller than that of kaons and thus that of strange and antistrange quarks.

\section{A. The dynamical coalescence model}

In the dynamical quark coalescence model, the probability for producing a hadron from partons in the QGP is given by the overlap of parton phase-space distributions at freeze-out with the parton Wigner phase-space function inside the hadron. For a QGP containing $A$ partons, the momentum distribution of a $M$-parton hadron can be expressed as [56,57]

$$
\begin{aligned}
\frac{d N_{M}}{d^{3} K}= & G\left(\begin{array}{c}
A \\
M
\end{array}\right) \frac{1}{A^{M}} \int \prod_{i=1}^{M} f_{i}\left(\mathbf{r}_{i}, \mathbf{k}_{i}\right) \\
& \times \rho^{W}\left(\mathbf{r}_{i_{1}}, \mathbf{k}_{i_{1}} \cdots \mathbf{r}_{i_{M-1}}, \mathbf{k}_{i_{M-1}}\right) \\
& \times \delta\left[\mathbf{K}-\left(\mathbf{k}_{1}+\cdots+\mathbf{k}_{M}\right)\right] d \mathbf{r}_{1} d \mathbf{k}_{1} \cdots d \mathbf{r}_{M} d \mathbf{k}_{M},
\end{aligned}
$$

where $f_{i}$ is the parton phase-space distribution functions at freeze-out; $\rho^{W}$ is the $M$-parton Wigner phase-space function inside the hadron; $\mathbf{r}_{i_{1}}, \ldots, \mathbf{r}_{i_{M-1}}$ and $\mathbf{k}_{i_{1}}, \ldots, \mathbf{k}_{i_{M-1}}$ are, respectively, the $M-1$ relative coordinates and momenta taken at equal time in the $M$-parton rest frame $(\mathbf{K}=\mathbf{0})$; and $G$ is the statistical factor for the $M$ partons to form the hadron.

In transport model simulations of heavy-ion collisions, the multiplicity of a $M$-parton hadron produced from the dynamical quark coalescence model is then given by [56,57]

$$
\begin{aligned}
N_{M}= & G \int \sum_{i_{1}>i_{2}>\cdots>i_{M}} d \mathbf{r}_{i_{1}} d \mathbf{k}_{i_{1}} \cdots d \mathbf{r}_{i_{M-1}} d \mathbf{k}_{i_{M-1}} \\
& \times\left\langle\rho_{i}^{W}\left(\mathbf{r}_{i_{1}}, \mathbf{k}_{i_{1}} \cdots \mathbf{r}_{i_{M-1}}, \mathbf{k}_{i_{M-1}}\right)\right\rangle,
\end{aligned}
$$

where $\langle\cdots\rangle$ denotes event averaging and the sum runs over all possible combinations of $M$ partons.

To determine the statistical factor $G$ for the $\phi$ meson, we note that its quark wave function in the color-spin-isospin space can be expressed as a linear combination of all possible orthogonal flavor, color, and spin basis states [1,58]. The probability for a strange quark $s$ and an antistrange quark $\bar{s}$ to form a hadron with quantum numbers corresponding to a $\phi$ meson with the $z$ component of its spin equal to 1 is simply given by the probability of finding the two $s$ and $\bar{s}$ in any one of these color-spin-isospin basis states (i.e., $1 / 3^{2} \times 1 / 2^{2}=1 / 36$ ). Including also the possibility of forming a $\phi$ meson with the $z$ component of its spin equal to -1 and 0 triples the probability. As a result, the statistical factor $G$ for the $\phi$ meson is $1 / 12$. A similar consideration leads to a statistical factor $G=1 / 54$ for the $\Omega^{-}$and $\bar{\Omega}^{+}$baryons.

\section{B. Parton phase-space distributions}

The $M$-parton phase-space distribution function $\prod_{i=1}^{M} f\left(\mathbf{r}_{i}, \mathbf{k}_{i}\right)$ in Eq. (3) refers to partons with spatial coordinates and momenta at equal time in the rest frame of the $M$-parton cluster. Since parton momenta in the AMPT model are given in the nucleus-nucleus center-of-mass system, a Lorentz transformation is performed to obtain their momenta in the rest frame of the $M$-parton cluster. Furthermore, partons freeze out at different times as shown in Figs. 4 and 5. The same Lorentz transformation is thus used in obtaining their 
space-time coordinates in the rest frame of the $M$-parton cluster. To determine the spatial coordinates of these partons at equal time in their rest frame, partons in the cluster that freeze out earlier are allowed to propagate freely, that is, with constant velocities given by the ratio of their momenta and energies in the rest frame of the cluster, until the time when the last parton in the cluster freezes out.

\section{Quark Wigner phase-space functions inside $\phi$ and $\Omega$}

To determine the quark Wigner phase-space functions inside hadrons requires knowledge of their quark wave functions. For the $\phi$ meson, we take its quark wave function to be that of a spherical harmonic oscillator, that is,

$$
\psi\left(\mathbf{r}_{1}, \mathbf{r}_{2}\right)=1 /\left(\pi \sigma_{\phi}^{2}\right)^{3 / 4} \exp \left[-r^{2} /\left(2 \sigma_{\phi}^{2}\right)\right],
$$

in terms of the relative coordinate $\mathbf{r}=\mathbf{r}_{1}-\mathbf{r}_{2}$ and the size parameter $\sigma_{\phi}$. This normalized wave function leads to a root mean-square radius $R_{\phi}=\left\langle r^{2}\right\rangle^{1 / 2}=(3 / 8)^{1 / 2} \sigma_{\phi}$ for the $\phi$ meson.

The quark Wigner phase-space function inside the $\phi$ meson is obtained from its quark wave function by

$$
\begin{aligned}
\rho_{\phi}^{W}(\mathbf{r}, \mathbf{k}) & =\int \psi\left(\mathbf{r}+\frac{\mathbf{R}}{2}\right) \psi^{*}\left(\mathbf{r}-\frac{\mathbf{R}}{2}\right) \exp (-i \mathbf{k} \cdot \mathbf{R}) d^{3} \mathbf{R} \\
& =8 \exp \left(-\frac{r^{2}}{\sigma_{\phi}^{2}}-\sigma_{\phi}^{2} k^{2}\right),
\end{aligned}
$$

where $\mathbf{k}=\left(\mathbf{k}_{1}-\mathbf{k}_{2}\right) / 2$ is the relative momentum between $s$ and $\bar{s}$ quarks.

For $\Omega^{-}$and $\bar{\Omega}^{+}$baryons, their quark wave functions are taken to be the same and are given by that of a spherical harmonic oscillator as well $[59,60]$, that is,

$$
\psi\left(\mathbf{r}_{1}, \mathbf{r}_{2}, \mathbf{r}_{3}\right)=\left(3 \pi^{2} \sigma_{\Omega}^{4}\right)^{-3 / 4} \exp \left(-\frac{\rho^{2}+\lambda^{2}}{2 \sigma_{\Omega}^{2}}\right),
$$

in terms of the relative coordinates $\rho$ and $\lambda$ and the size parameter $\sigma_{\Omega}$. Here, we have used the usual Jacobi coordinates for a three-particle system [57], that is,

$$
\left(\begin{array}{l}
\mathbf{R} \\
\rho \\
\lambda
\end{array}\right)=\left(\begin{array}{ccc}
\frac{1}{3} & \frac{1}{3} & \frac{1}{3} \\
\frac{1}{\sqrt{2}} & -\frac{1}{\sqrt{2}} & 0 \\
\frac{1}{\sqrt{6}} & \frac{1}{\sqrt{6}} & -\frac{2}{\sqrt{6}}
\end{array}\right)\left(\begin{array}{l}
\mathbf{r}_{1} \\
\mathbf{r}_{2} \\
\mathbf{r}_{3}
\end{array}\right),
$$

where $\mathbf{R}$ is the center-of-mass coordinate of the three quarks or antiquarks.

Using $d \mathbf{r}_{1} d \mathbf{r}_{2} d \mathbf{r}_{3}=3^{3 / 2} d \mathbf{R} d \rho d \lambda$, it is easy to check that the wave function given by Eq. (7) is normalized to one. From the relation $\left(\mathbf{r}_{1}-\mathbf{R}\right)^{\mathbf{2}}+\left(\mathbf{r}_{2}-\mathbf{R}\right)^{\mathbf{2}}+\left(\mathbf{r}_{3}-\mathbf{R}\right)^{\mathbf{2}}=\rho^{\mathbf{2}}+\lambda^{\mathbf{2}}$, the root mean-square radius $R_{\Omega}$ of the $\Omega$ baryon is given by

$$
R_{\Omega}=\left[\int \frac{\rho^{2}+\lambda^{2}}{3}\left|\psi\left(\mathbf{r}_{1}, \mathbf{r}_{2}, \mathbf{r}_{3}\right)\right|^{2} 3^{3 / 2} d \rho d \lambda\right]^{1 / 2}=\sigma_{\Omega} .
$$

The quark Wigner phase-space function inside the $\Omega$ baryon is obtained from its quark wave function via

$$
\begin{aligned}
& \rho_{\Omega}^{W}\left(\rho, \lambda, \mathbf{k}_{\rho}, \mathbf{k}_{\lambda}\right) \\
& =\int \psi\left(\rho+\frac{\mathbf{R}_{\mathbf{1}}}{2}, \lambda+\frac{\mathbf{R}_{\mathbf{2}}}{2}\right) \psi^{*}\left(\rho-\frac{\mathbf{R}_{\mathbf{1}}}{2}, \lambda-\frac{\mathbf{R}_{\mathbf{2}}}{2}\right)
\end{aligned}
$$

$$
\begin{aligned}
& \times \exp \left(-i \mathbf{k}_{\rho} \cdot \mathbf{R}_{\mathbf{1}}\right) \exp \left(-i \mathbf{k}_{\lambda} \cdot \mathbf{R}_{\mathbf{2}}\right) 3^{3 / 2} d \mathbf{R}_{\mathbf{1}} d \mathbf{R}_{\mathbf{2}} \\
= & 8^{2} \exp \left(-\frac{\rho^{\mathbf{2}}+\lambda^{\mathbf{2}}}{\sigma_{\Omega}^{2}}\right) \exp \left[-\left(\mathbf{k}_{\rho}^{2}+\mathbf{k}_{\lambda}^{2}\right) \sigma_{\Omega}^{2}\right]
\end{aligned}
$$

where $\mathbf{k}_{\rho}$ and $\mathbf{k}_{\lambda}$ are relative momenta, which together with the total momentum $\mathbf{K}$ are defined by [57]

$$
\left(\begin{array}{c}
\mathbf{K} \\
\mathbf{k}_{\rho} \\
\mathbf{k}_{\lambda}
\end{array}\right)=\left(\begin{array}{ccc}
1 & 1 & 1 \\
\frac{1}{\sqrt{2}} & -\frac{1}{\sqrt{2}} & 0 \\
\frac{1}{\sqrt{6}} & \frac{1}{\sqrt{6}} & -\frac{2}{\sqrt{6}}
\end{array}\right)\left(\begin{array}{l}
\mathbf{k}_{1} \\
\mathbf{k}_{2} \\
\mathbf{k}_{3}
\end{array}\right),
$$

with $\mathbf{k}_{1}, \mathbf{k}_{2}$, and $\mathbf{k}_{3}$ being the momenta of the three quarks.

\section{D. $\phi$ and $\Omega$ size parameters}

The two parameters $\sigma_{\phi}$ and $\sigma_{\Omega}$ in the quark Wigner phasespace functions inside the $\phi$ meson and $\Omega$ baryon are related to their root-mean-square radii. Since the latter are not known empirically, we take them as adjustable parameters and fix them by fitting measured yields of $\phi$ mesons and $\Omega$ baryons in relativistic heavy-ion collisions.

For $\phi$ mesons, experimental data on their rapidity density $d N / d y$ at midrapidity and its centrality dependence are available for $\mathrm{Au}+\mathrm{Au}$ collisions at $\sqrt{s_{N N}}=200 \mathrm{GeV}[38,39]$. There is, however, a significant difference between the STAR and PHENIX data for the $\phi$ meson yield. For instance, the $\phi$ meson $d N / d y$ at midrapidity is about 7.7 in the STAR data but is 4.5 in the PHENIX data for $\mathrm{Au}+\mathrm{Au}$ collisions at $\sqrt{s_{N N}}=200 \mathrm{GeV}$ with a centrality of $0 \%-5 \%$. Using the dynamical quark coalescence model described here, we find that the STAR (PHENIX) data can be approximately reproduced with a $\phi$ meson size parameter $\sigma_{\phi} \approx 1.06(0.77) \mathrm{fm}$, which gives a reasonable $\phi$ meson root-mean-square radius of about $R_{\phi}=0.65(0.47) \mathrm{fm}$. For the impact parameter $b=8 \mathrm{fm}$ as mainly considered in the present study, the AMPT model gives the number of participant nucleons $N_{\text {part }} \approx 160$, which corresponds to a centrality of about $31 \%$. The resulting $\phi$ meson rapidity density $d N / d y$ at midrapidity obtained with the $\phi$ meson radius $R_{\phi}=0.65(0.47) \mathrm{fm}$ is about 3.3 (2.0), which is also in good agreement with experimental data $[38,39]$.

For $\Omega$ baryons, there are only preliminary experimental data on their $d N / d y$ at midrapidity in central collisions of $\mathrm{Au}+\mathrm{Au}$ at $\sqrt{s_{N N}}=200 \mathrm{GeV}$, and its value is about 0.64 [40]. Fitting the result from the dynamical quark coalescence model for the same collision at $b=0 \mathrm{fm}$ leads to an $\Omega$ baryon size parameter $\sigma_{\Omega} \approx 1.2 \mathrm{fm}$, corresponding to an $\Omega$ baryon root-mean-square radius of about $R_{\Omega} \approx 1.2 \mathrm{fm}$, which is somewhat large but not unrealistic. For more peripheral collisions at impact parameter $b=8 \mathrm{fm}$, an $\Omega$ radius of $R_{\Omega}=1.2 \mathrm{fm}$ leads to a rapidity density $d N / d y$ of about 0.26 at midrapidity.

In the following, we use these $\phi$ and $\Omega$ root-mean-square radii, that is, $R_{\phi}=0.65$ or $0.47 \mathrm{fm}$ for the $\phi$ meson and $R_{\Omega}=$ $1.2 \mathrm{fm}$ for the $\Omega$ baryon unless stated otherwise.

\section{IV. $\phi$ AND $\Omega$ PRODUCTION IN AU + AU COLLISIONS AT $\sqrt{S_{N N}}=200 \mathrm{GeV}$}

Using the phase-space information and the dynamical coalescence model described here, we can now study the 
transverse-momentum spectra of $\phi$ mesons and $\Omega$ baryons as well as their anisotropic flows in relativistic heavy-ion collisions.

\section{A. Transverse-momentum spectra of $\phi$ and $\Omega$}

Figure 8 shows the transverse-momentum spectra of midrapidity $\phi$ mesons and $\Omega$ baryons, as well as their ratio in $\mathrm{Au}+\mathrm{Au}$ collisions at $\sqrt{s_{N N}}=200 \mathrm{GeV}$ with $b=0 \mathrm{fm}$ (left panels) and $b=8 \mathrm{fm}$ (right panels), and $R_{\phi}=0.65$ or $0.47 \mathrm{fm}$ and $R_{\Omega}=1.2 \mathrm{fm}$. For comparison, we also include in the upper left panel of Fig. 8 the experimental data for $\phi$ mesons in the $0 \%-5 \%$ centrality bin from the STAR Collaboration [38] (solid stars) and in the $0 \%-10 \%$ centrality bin from the PHENIX Collaboration [39] (open stars) as well as the preliminary data for $\Omega$ baryons in the $0 \%-10 \%$ centrality bin from the STAR Collaboration [40] (solid triangles). Also shown in the lower left panel by open triangles are data on the $\Omega / \phi$ ratio in the $0 \%-10 \%$ centrality bin, obtained from a linear interpolation of the $\phi$ meson data in the upper left panel.

It is seen from the upper left panel of Fig. 8 that the calculated transverse-momentum spectrum of $\Omega$ baryons is stiffer than that of $\phi$ mesons, as expected from their different masses. The calculated transverse-momentum spectra of both $\phi$ mesons and $\Omega$ baryons are, however, significantly softer than the data. With $R_{\phi}=0.65(0.47) \mathrm{fm}$, the mean transverse-momentum calculated from the $\phi$ meson transverse-momentum spectrum is about $0.72(0.73)$ and

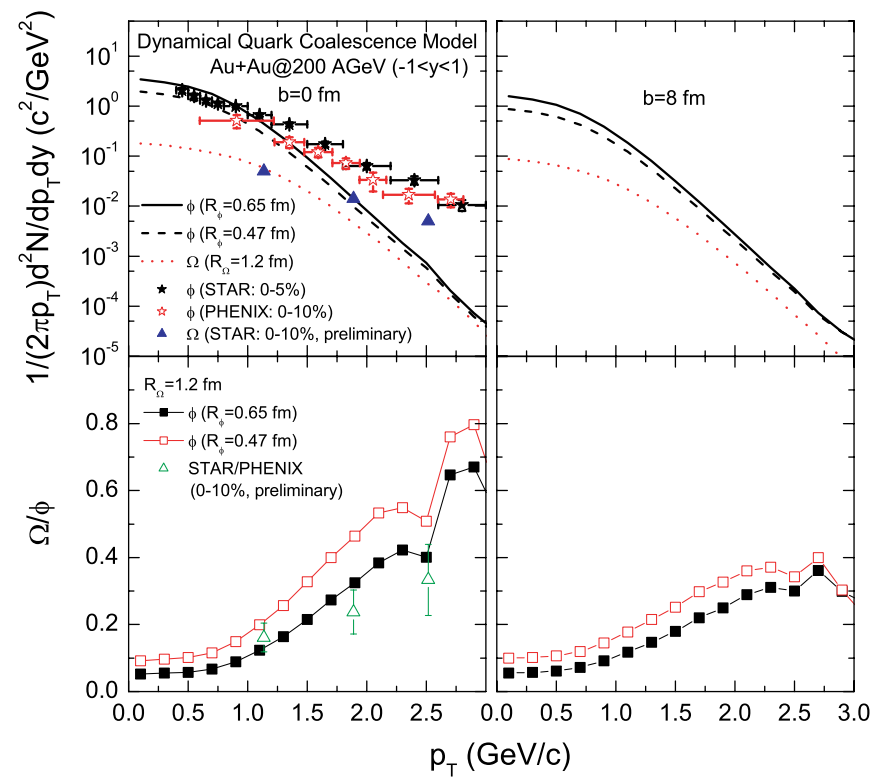

FIG. 8. (Color online) Transverse-momentum dependence of midrapidity $\phi$ mesons and $\Omega\left(\Omega^{-}+\bar{\Omega}^{+}\right)$baryons (upper panels) and their ratio (lower panels) in $\mathrm{Au}+\mathrm{Au}$ collisions at $\sqrt{s_{N N}}=200 \mathrm{GeV}$ with $b=0 \mathrm{fm}$ (left panels) and $b=8 \mathrm{fm}$ (right panels). Data for $\phi$ mesons in the $0 \%-5 \%$ centrality bin from the STAR Collaboration [38] (solid stars) and in the 0\%-10\% centrality bin from the PHENIX Collaboration [39] (open stars) as well as preliminary data for $\Omega$ baryons in 0\%-10\% centrality bin from the STAR Collaboration [40] (solid triangles) are shown in the upper left panel; those for the $\Omega / \phi$ ratio in the $0 \%-10 \%$ centrality bin are shown in the lower left panel.
$0.68(0.70) \mathrm{GeV} / c$ for $b=0 \mathrm{fm}$ and $b=8 \mathrm{fm}$, respectively, compared with the experimental value of about $0.85-1.1 \mathrm{GeV} / c[38,39]$. For $\Omega$ baryons, their mean transverse momentum is about 0.93 and $0.83 \mathrm{GeV} / c$ for $b=0 \mathrm{fm}$ and $b=8 \mathrm{fm}$, respectively, and are larger than those of $\phi$ mesons.

We note that hadron transverse-momentum spectra obtained from the AMPT model with string melting are generally softer than those measured in experiments [61]. Since hadron spectra reflect those of partons in the parton coalescence model, soft hadron spectra are due to soft parton spectra during the partonic stage. As discussed in Ref. [61], the latter is a result of the small current quark masses used in the parton cascade of the AMPT model with string melting, which make their transverse-momentum spectra less affected by radial collective flow than hadrons in the default AMPT model. Since anisotropic flows are given by ratios of hadron transverse-momentum spectra, predictions of anisotropic flows as well as the ratio of hadron transverse-momentum spectra from the present AMPT model are expected to be more reliable $[18,61]$.

The ratio of midrapidity $\Omega$ baryons to $\phi$ mesons shown in the lower panels of Fig. 8 is seen to increase appreciably from low $p_{T}$ to intermediate $p_{T}$ of about $2.5 \mathrm{GeV} / \mathrm{c}$. The enhancement factor is about 7 and 4 for $b=0 \mathrm{fm}$ and $b=8 \mathrm{fm}$, respectively. This enhancement is a result of parton coalescence as in the case of observed anomalously large antiproton to pion ratios of about 0.8 and 0.4 at $p_{T} \gtrsim 2.5 \mathrm{GeV} / c$ in central and midperipheral (centrality of about $30 \%$ ) $\mathrm{Au}+\mathrm{Au}$ collisions at $\sqrt{s_{N N}}=200 \mathrm{GeV}$, respectively [62]. As explained in Refs. [1-3], in the coalescence model baryons with transverse momentum $p_{T}$ are mainly formed from quarks with transverse momenta $\sim p_{T} / 3$, whereas mesons with the same transverse momentum are mainly produced from partons with transverse momenta $\sim p_{T} / 2$. Since quark transverse-momentum spectra decrease with $p_{T}$, it is more favorable to produce high-transverse-momentum baryons than mesons if hadrons are produced from the QGP through the coalescence of quarks. However, the $\bar{\Omega}^{+} / \phi$ ratio is only about 0.23 and 0.17 at $p_{T} \approx 2.5 \mathrm{GeV} / c$ for $b=0 \mathrm{fm}$ and $b=8 \mathrm{fm}$, respectively, and is significantly smaller than the anti-proton to pion ratio observed in experiments. For $b=0 \mathrm{fm}$, our result with $R_{\phi}=0.65 \mathrm{fm}$ reproduces reasonably the measured $\Omega / \phi$ ratio as shown in the lower left panel of Fig. 8.

In Fig. 9, we show the $p_{T}$ dependence of the ratio of midrapidity $\phi$ meson or $\Omega$ baryon yield in central $(b=0 \mathrm{fm})$ to the corresponding midperipheral $(b=8 \mathrm{fm})$ collisions of $\mathrm{Au}+\mathrm{Au}$ at $\sqrt{s_{N N}}=200 \mathrm{GeV}$. It is seen that the $p_{T}$ dependence is very different for $\phi$ mesons and $\Omega$ baryons with the latter having a smaller value at low $p_{T}$ while a larger value at high $p_{T}$ than for $\phi$ mesons. Our results thus indicate that nuclear suppression at intermediate momenta is weaker for $\Omega$ baryons than for $\phi$ mesons. This feature is consistent with the measured $p_{T}$ dependence of so-called $\mathrm{R}_{\mathrm{CP}}$ (the ratio of binary-collision-scaled $p_{T}$ spectra in central and peripheral collisions) for $p+\bar{p}$ and pions [62].

\section{B. Anisotropic flows of $\phi$ and $\Omega$}

In heavy-ion collisions at RHIC, experimental data have indicated that there exist not only strong elliptic flow but also a clear fourth-order anisotropic flow $v_{4}$ for charged hadrons 


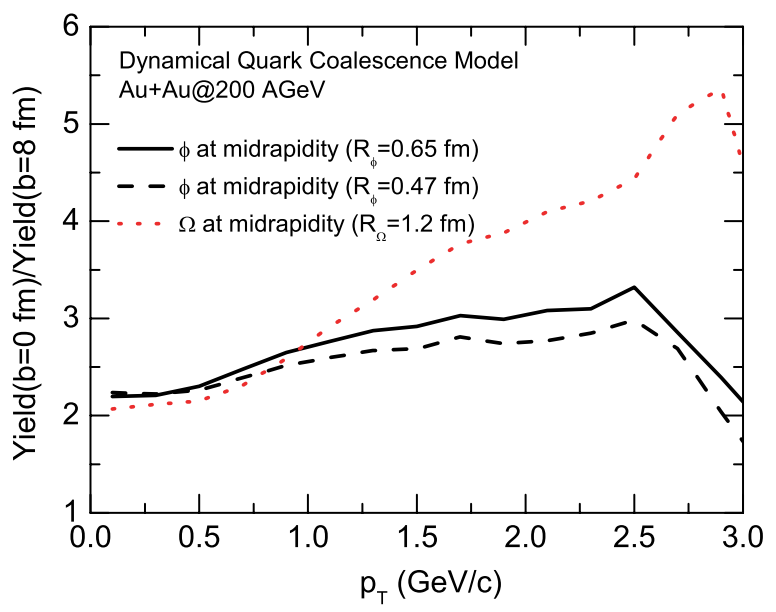

FIG. 9. (Color online) Transverse-momentum dependence of the ratio of midrapidity $\phi$ meson and $\Omega$ baryon yields in central $(b=$ $0 \mathrm{fm})$ to that in midperipheral $(b=8 \mathrm{fm}) \mathrm{Au}+\mathrm{Au}$ collisions at $\sqrt{s_{N N}}=200 \mathrm{GeV}$.

[26,27]. An interesting finding about $v_{4}$ is that it scales with the square of $v_{2}$ [i.e., $v_{4}\left(p_{T}\right) \sim v_{2}^{2}\left(p_{T}\right)$ ]. In the present section, both elliptic and fourth-order anisotropic flows of $\phi$ mesons and $\Omega$ baryons are studied to see if they also show similar features.

In the left panel of Fig. 10, we show the $p_{T}$ dependence of anisotropic flows $v_{2}$ and $v_{4}$ of midrapidity $\phi$ mesons produced in $\mathrm{Au}+\mathrm{Au}$ collisions at $\sqrt{s_{N N}}=200 \mathrm{GeV}$ and $b=8 \mathrm{fm}$. Solid and open squares are, respectively, the $\phi$ meson $v_{2}$ and $v_{4}$ for a $\phi$ meson radius $R_{\phi}=0.65 \mathrm{fm}$; solid and open triangles are, respectively, those for $R_{\phi}=0.47 \mathrm{fm}$. It is seen that the scaling relation $v_{4}\left(p_{T}\right) \sim v_{2}^{2}\left(p_{T}\right)$ is satisfied in both cases, as shown by the solid $\left(1.1 v_{2}^{2}\right)$ and dashed $\left(1.2 v_{2}^{2}\right)$ lines in the same figure. It is interesting to see that the scaling

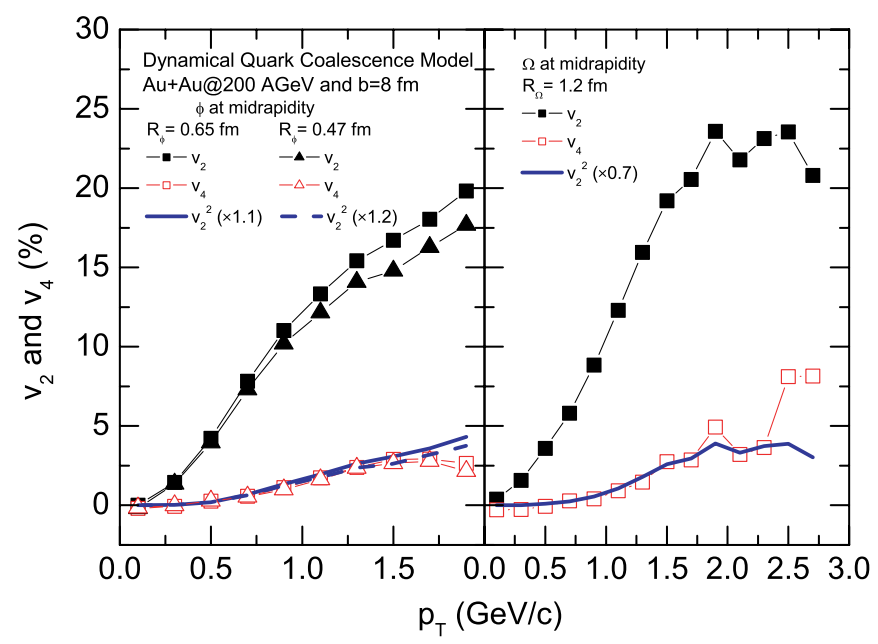

FIG. 10. (Color online) Transverse-momentum dependence of anisotropic flows $v_{2}$ and $v_{4}$ of midrapidity $\phi$ mesons (left panel) and $\Omega$ baryons (right panel) produced in $\mathrm{Au}+\mathrm{Au}$ collisions at $\sqrt{s_{N N}}=200 \mathrm{GeV}$ and $b=8 \mathrm{fm}$. Solid and dashed lines in the left panel are, respectively, $1.1 v_{2}^{2}$ and $1.2 v_{2}^{2}$ for $\phi$ mesons; the solid line in the right panel is $0.7 v_{2}^{2}$ for $\Omega$ baryons. coefficient of 1.1 or 1.2 is the same as that extracted from the data for charged hadrons [26,27]. Similar results for $\Omega$ baryons using the radius $R_{\Omega}=1.2 \mathrm{fm}$ are shown in the right panel of Fig. 10. The scaling relation $v_{4}\left(p_{T}\right) \sim v_{2}^{2}\left(p_{T}\right)$ is again satisfied for $\Omega$ baryons but with a smaller scaling coefficient than that for $\phi$ mesons, as shown by the solid line $\left(0.7 v_{2}^{2}\right)$ in the right panel.

Based on the naive momentum-space quark coalescence model that only allows quarks with equal momentum to form hadrons [4], this scaling relation between hadron $v_{2}\left(p_{T}\right)$ and $v_{4}\left(p_{t}\right)$ has been attributed to a similar scaling relation between those of quarks $[28,29]$. By neglecting the small contribution of higher order anisotropic flows (higher than fourth order), this model gives for midrapidity hadrons

$$
\begin{aligned}
\frac{v_{4, M}\left(2 p_{T}\right)}{v_{2, M}^{2}\left(2 p_{T}\right)} & \approx \frac{1}{4}+\frac{1}{2} \frac{v_{4, q}\left(p_{T}\right)}{v_{2, q}^{2}\left(p_{T}\right)}, \\
\frac{v_{4, B}\left(3 p_{T}\right)}{v_{2, B}^{2}\left(3 p_{T}\right)} & \approx \frac{1}{3}\left(1+\frac{v_{4, q}\left(p_{T}\right)}{v_{2, q}^{2}\left(p_{T}\right)}\right),
\end{aligned}
$$

where $v_{n, M}\left(p_{T}\right), v_{n, B}\left(p_{T}\right)$, and $v_{n, q}\left(p_{T}\right)$ denote, respectively, the meson, baryon, and quark anisotropic flows. The hadron anisotropic flows thus satisfy the scaling relation $v_{4}\left(p_{T}\right) \sim$ $v_{2}^{2}\left(p_{T}\right)$ if a similar scaling relation is satisfied by quark anisotropic flows.

We have already shown in Fig. 7 that the scaling relation $v_{4}\left(p_{T}\right) \sim v_{2}^{2}\left(p_{T}\right)$ is satisfied by midrapidity strange and antistrange quarks $(s+\bar{s})$ with a scaling coefficient of about 0.85 [i.e., $v_{4, q}\left(p_{T}\right) / v_{2, q}^{2}\left(p_{T}\right) \approx 0.85$ ]. Equations $(12)$ then lead to a scaling coefficient of $v_{4, \phi}\left(p_{T}\right) / v_{2, \phi}^{2}\left(p_{T}\right) \approx 0.68$ for $\phi$ mesons and $v_{4, \Omega}\left(p_{T}\right) / v_{2, \Omega}^{2}\left(p_{T}\right) \approx 0.62$ for $\Omega$ baryons. Compared with the scaling coefficients of 1.1 or 1.2 for $\phi$ mesons and 0.7 for $\Omega$ baryons from the dynamical quark coalescence model, the predicted value from the naive momentum-space quark coalescence model for the $\phi$ meson scaling coefficient is significantly smaller. However, the dynamical quark coalescence model does give a larger scaling coefficient for $\phi$ mesons than for $\Omega$ baryons, as expected from the naive momentum-space coalescence model.

Another interesting and important finding in heavy-ion collisions at RHIC is the valence quark number scaling of the elliptic flow of identified hadrons. That is, the elliptic flow per valence quark in a hadron is the same at the same transverse momentum per valence quark [i.e., $v_{2, H}\left(p_{T} / n_{q}\right) / n_{q}=$ $v_{2, q}\left(p_{T}\right)$ with $v_{2, H}$ and $n_{q}$ denoting, respectively, the hadron $v_{2}$ and the number of valence quarks or antiquarks in a hadron]. In the naive momentum-space quark coalescence model, this scaling has been shown to be satisfied if high-order anisotropic flows are small $[4,29]$. The scaling is, however, broken in more general quark coalescence models that take into account the quark momentum distribution [63-65] and higher parton Fock states [66] in hadrons as well as the effect of resonance decays [63]. It is thus of interest to see if the elliptic flows of $\phi$ mesons and $\Omega$ baryons from the present dynamical quark coalescence model also satisfy the valence quark number scaling.

In Fig. 11, we show the valence quark number scaled elliptic flow $v_{2} / n_{q}$ as a function of scaled transverse momentum 


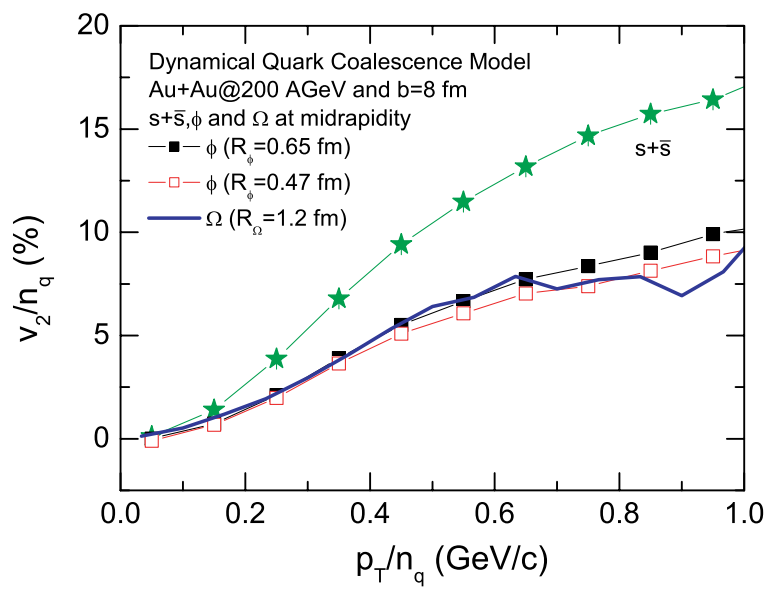

FIG. 11. (Color online) Valence quark number scaled elliptic flow $v_{2} / n_{q}$ as a function of scaled transverse momentum $p_{T} / n_{q}$ for midrapidity $\phi$ mesons and $\Omega$ baryons produced in $\mathrm{Au}+\mathrm{Au}$ collisions at $\sqrt{s_{N N}}=200 \mathrm{GeV}$ and $b=8 \mathrm{fm}$ with $R_{\phi}=0.65 \mathrm{fm}$ (solid squares) and $0.47 \mathrm{fm}$ (open squares) as well as $R_{\Omega}=1.2 \mathrm{fm}$ (solid line). Results for midrapidity strange and antistrange quarks $(s+\bar{s})$ at freeze-out are shown by solid stars.

$p_{T} / n_{q}$ for midrapidity $\phi$ mesons and $\Omega$ baryons produced in $\mathrm{Au}+\mathrm{Au}$ collisions at $\sqrt{s_{N N}}=200 \mathrm{GeV}$ and $b=8 \mathrm{fm}$ with $R_{\phi}=0.47$ or $0.65 \mathrm{fm}$ and $R_{\Omega}=1.2 \mathrm{fm}$. For comparison, we also include the elliptic flow of midrapidity strange and antistrange quarks $(s+\bar{s})$ at freeze-out. It is seen that the elliptic flows of both midrapidity $\phi$ mesons and $\Omega$ baryons from the dynamical quark coalescence model satisfy the valence quark number scaling. The valence quark number scaled elliptic flows of $\phi$ mesons and $\Omega$ baryons are, however, significantly smaller than that of coalescing strange and antistrange quarks. This feature is different from the prediction of the naive momentum-space quark coalescence model where the valence quark number scaled $v_{2}$ of hadrons is equal to the $v_{2}$ of coalescing quarks. Our results therefore indicate that both the dynamical phase-space information of quarks and the quark phase-space distribution inside hadrons play important roles in hadron anisotropic flows, and cautions are needed in interpreting the elliptic flow of partons from that of hadrons using the naive momentum-space parton coalescence model. We note that our results are consistent with previous studies on $v_{2}$ of protons and pions based on the parton phase-space information from Molnar's parton cascade (MPC) model [5].

\section{HADRON SIZE DEPENDENCE OF $\phi$ AND $\Omega$ YIELDS AND ANISOTROPIC FLOWS}

In all of our calculations, we have used $\phi$ meson and $\Omega$ baryon size parameters that are fixed from fitting measured yields in experiments. Ideally, we would like to use the empirically and/or theoretically determined root-mean-square radii of the $\phi$ meson and the $\Omega$ baryon in the dynamical quark coalescence model. In the absence of such information, it is of interest to study how our results depend on the sizes of the $\phi$ meson and the $\Omega$ baryon.

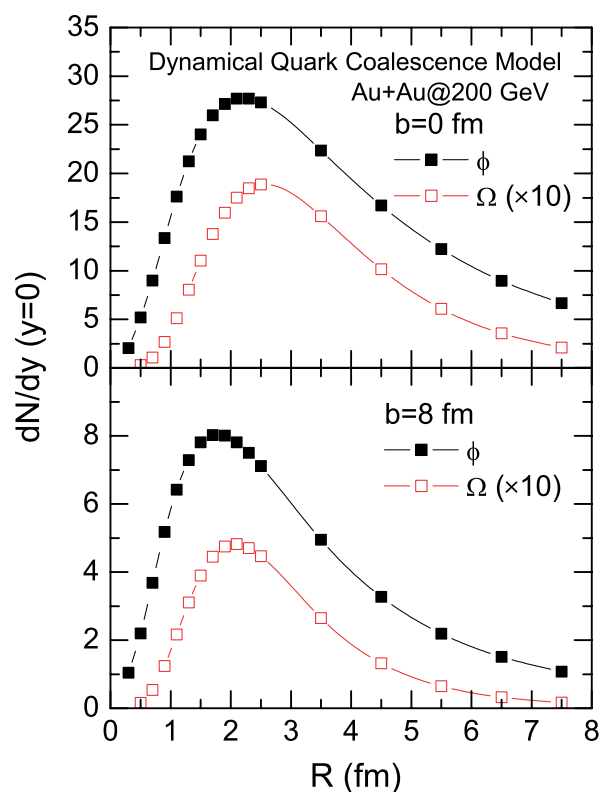

FIG. 12. (Color online) Hadron size (root-mean-square radius) dependence of rapidity density $d N / d y$ of midrapidity $\phi$ mesons (solid squares) and $\Omega\left(\Omega^{-}+\bar{\Omega}^{+}\right)$baryons (open squares) produced in $\mathrm{Au}+\mathrm{Au}$ collisions at $\sqrt{s_{N N}}=200 \mathrm{GeV}$ with $b=0 \mathrm{fm}$ (upper panel) and $b=8 \mathrm{fm}$ (lower panel).

\section{A. Hadron size dependence of $\phi$ and $\Omega$ yields}

We show in Fig. 12 the rapidity density $d N / d y$ of midrapidity $\phi$ mesons and $\Omega$ baryons produced in $\mathrm{Au}+\mathrm{Au}$ collisions at $\sqrt{s_{N N}}=200 \mathrm{GeV}$ with $b=0 \mathrm{fm}$ (upper panel) and $b=8 \mathrm{fm}$ (lower panel) as functions of their root-mean-square radii. It is seen that both $\phi$ meson and $\Omega$ baryon yields are sensitive to their sizes. For physically reasonable radii, both yields increase with increasing radii but decrease eventually as the radii become unrealistically large. For collisions at impact parameter $b=0 \mathrm{fm}$, the yield exhibits maxima at $R_{\phi}=2.2 \mathrm{fm}$ for $\phi$ mesons and at $R_{\Omega}=2.5 \mathrm{fm}$ for $\Omega$ baryons. A similar feature is observed in the case of $b=8 \mathrm{fm}$ but the maximum is at $R_{\phi}=1.75 \mathrm{fm}$ for $\phi$ mesons and at $R_{\Omega}=2 \mathrm{fm}$ for $\Omega$ baryons.

\section{B. Hadron size dependence of $\phi$ and $\Omega$ elliptic flows and the valence quark number scaling}

We first show in the left panel of Fig. 13 the $p_{T}$ dependence of the $v_{2}$ of midrapidity $\phi$ mesons in $\mathrm{Au}+\mathrm{Au}$ collisions at $\sqrt{s_{N N}}=200 \mathrm{GeV}$ and $b=8 \mathrm{fm}$ for different values of root-mean-square radius $R_{\phi}$. It is clearly seen that the $v_{2}$ of $\phi$ mesons depends strongly on the $\phi$ meson size. It becomes larger with increasing $R_{\phi}$ but changes little when $R_{\phi}>4.5 \mathrm{fm}$. Similarly, we show in the right panel of Fig. 13 the $p_{T}$ dependence of the $v_{2}$ of midrapidity $\Omega$ baryons produced in the same collision with different values of $R_{\Omega}$. Similarly to that of $\phi$ mesons, the $v_{2}$ of $\Omega$ baryons also increases with increasing size of $\Omega$ baryon and saturates when $R_{\Omega}>4.5 \mathrm{fm}$. The saturation of $\phi$ meson and $\Omega$ baryon $v_{2}$ at large sizes observed in Fig. 13 occurs essentially because the dynamical quark 


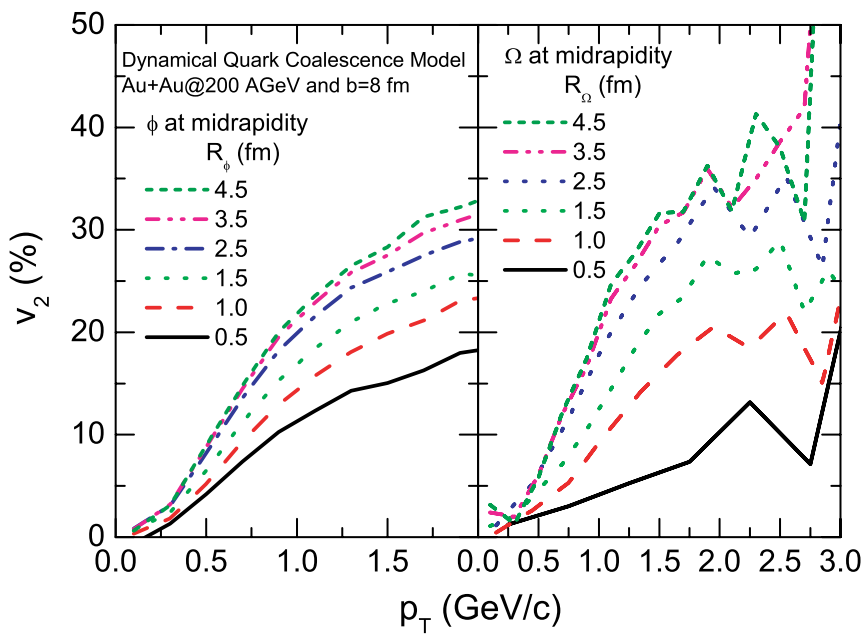

FIG. 13. (Color online) Transverse-momentum dependence of the $v_{2}$ of midrapidity $\phi$ mesons (left panel) and $\Omega$ baryons (right panel) in $\mathrm{Au}+\mathrm{Au}$ collisions at $\sqrt{s_{N N}}=200 \mathrm{GeV}$ and $b=8 \mathrm{fm}$ with different values of root-mean-square radii $R_{\phi}$ and $R_{\Omega}$.

coalescence model approaches the naive momentum-space quark coalescence model (i.e, only partons with the same momentum can coalesce into hadrons, when the hadron size is large enough). This can be easily seen from Eqs. (6) and (10) as the quark Wigner phase-space function becomes essentially delta functions in the relative momenta of coalescing partons when hadron sizes (i.e., the parameters $\sigma_{\phi}$ and $\sigma_{\Omega}$ ) are very large. As a result, we expect that the valence quark number scaled elliptic flows of $\phi$ mesons and $\Omega$ baryons should approach the $v_{2}$ of coalescing strange and antistrange quarks as their sizes increase. This is shown in Fig. 14, where it is seen that the $p_{T} / n_{q}$ dependence of $v_{2} / n_{q}$ for midrapidity $\phi$ mesons and $\Omega$ baryons produced in $\mathrm{Au}+\mathrm{Au}$ collisions at $\sqrt{s_{N N}}=200 \mathrm{GeV}$ and $b=8 \mathrm{fm}$ with $R_{\phi}=R_{\Omega}=4.5 \mathrm{fm}$ is indeed similar to the elliptic flow of midrapidity strange and antistrange quarks $(s+\bar{s})$ at freeze out.

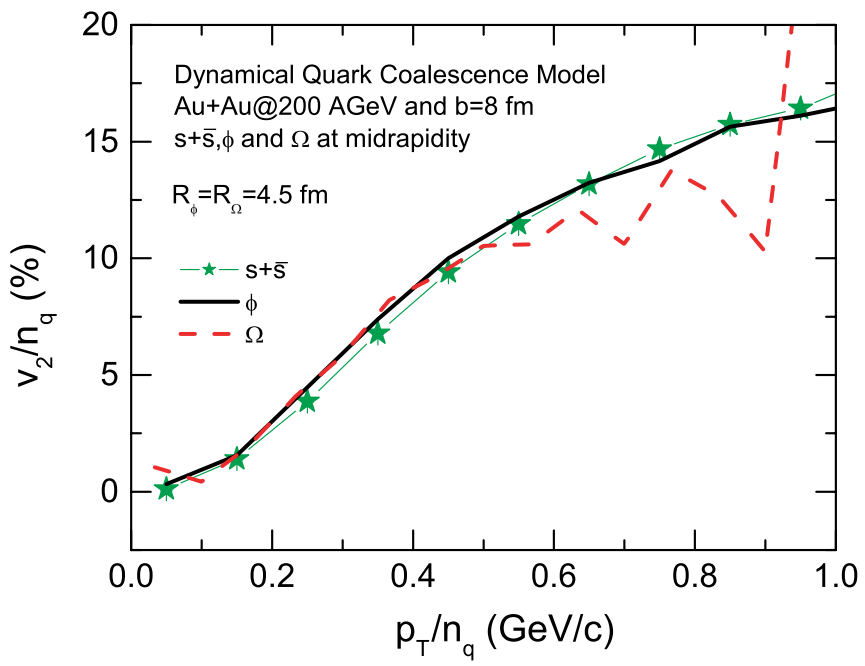

FIG. 14. (Color online) Same as Fig. 11 but with $R_{\phi}=R_{\Omega}=$ $4.5 \mathrm{fm}$.

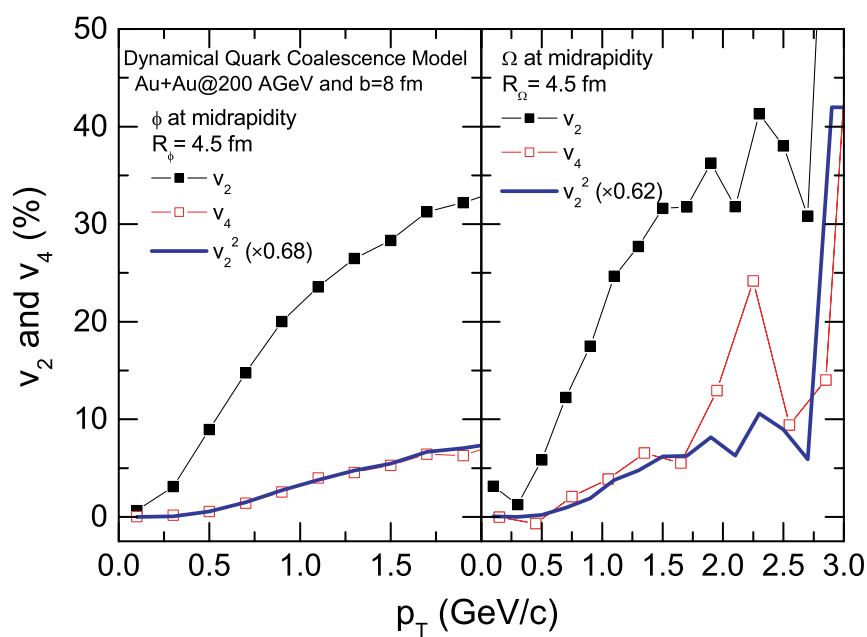

FIG. 15. (Color online) Same as Fig. 10 but with $R_{\phi}=R_{\Omega}=$ $4.5 \mathrm{fm}$.

\section{Fourth-order flows of $\phi$ and $\Omega$}

For the scaling relation $v_{4}\left(p_{T}\right) \sim v_{2}^{2}\left(p_{T}\right)$ among hadron anisotropic flows, as discussed here and in Refs. [28,29], the dynamical quark coalescence model leads to a deviation of the scaling coefficient from that of the naive momentum-space quark coalescence model as a result of finite hadron sizes and nonzero parton relative momenta inside hadrons. The deviation is expected to disappear for large enough hadron sizes when the dynamical quark coalescence model approaches the naive momentum-space quark coalescence model. This is demonstrated in Fig. 15, where we show the $p_{T}$ dependence of the anisotropic flows $v_{2}$ (solid squares) and $v_{4}$ (open squares) of midrapidity $\phi$ mesons (left panel) and $\Omega$ baryons (right panel) produced in $\mathrm{Au}+\mathrm{Au}$ collisions at $\sqrt{s_{N N}}=200 \mathrm{GeV}$ and $b=8 \mathrm{fm}$ with large hadron sizes (i.e., $R_{\phi}=R_{\Omega}=4.5 \mathrm{fm}$ ). Also shown by solid lines are $0.68 v_{2}^{2}$ for $\phi$ mesons in the left panel and $0.62 v_{2}^{2}$ for $\Omega$ baryons in the right panel. It is seen that for such large hadron sizes the scaling coefficients indeed approach the values expected from the naive momentum-space quark coalescence model (i.e., about 0.68 for $\phi$ mesons and 0.62 for $\Omega$ baryons). We note that a larger hadron size generally gives a smaller scaling coefficient in the dynamical quark coalescence model.

\section{QUARK MASS DEPENDENCE OF $\phi$ AND $\Omega$ TRANSVERSE-MOMENTUM SPECTRA AND ANISOTROPIC FLOWS}

The previous results were obtained with the current strange quark mass of $199 \mathrm{MeV}$, which is the default value in the AMPT model with string melting. Usually, the constituent quark mass (about $500 \mathrm{MeV}$ for the strange quark) is used in the quark coalescence model [1-4], so the binding energy effect can be neglected. In the following, we study the dependence of $\phi$ meson and $\Omega$ baryon production and their anisotropic flows on the strange quark mass. In particular, we use the constituent strange quark mass of $500 \mathrm{MeV}$ but the same strange quark space-time coordinates and momenta at freeze-out as before. The quark mass effect thus shows up through the Lorentz 
transformation that changes the strange quark space-time coordinates and momenta in the rest frame of the $\phi$ meson and the $\Omega$ baryon and therefore affects their overlap with the quark Wigner phase-space functions inside hadrons.

\section{A. Yields and transverse-momentum spectra of $\phi$ and $\Omega$}

Using a strange quark mass of $500 \mathrm{MeV}$, we find that yields of both $\phi$ mesons and $\Omega$ baryons are enhanced significantly compared to previous results with a strange quark mass of $199 \mathrm{MeV}$. For $\mathrm{Au}+\mathrm{Au}$ collisions at $\sqrt{s_{N N}}=200 \mathrm{GeV}$ and $b=0(8) \mathrm{fm}$, the $d N / d y$ at midrapidity for $\phi$ mesons with $R_{\phi}=0.65 \mathrm{fm}$ changes from 8.0 (3.3) to 12.2 (4.8) whereas that with $R_{\phi}=0.47 \mathrm{fm}$ changes from 4.7 (2.0) to 7.3 (3.0) when the strange quark mass changes from 199 to $500 \mathrm{MeV}$. To keep the $d N / d y$ of $\phi$ mesons at midrapidity unchanged requires a reduction of the $\phi$ meson size from $R_{\phi}=0.65$ to $0.5 \mathrm{fm}$ or from $R_{\phi}=0.47$ to $0.35 \mathrm{fm}$ when the strange quark mass is increased to $500 \mathrm{MeV}$. For $\Omega$ baryons, the value of $d N / d y$ at midrapidity for $b=0$ (8) fm with $R_{\Omega}=1.2 \mathrm{fm}$ changes from $0.65(0.26)$ to $1.36(0.49)$ when the strange quark mass changes from 199 to $500 \mathrm{MeV}$. A somewhat smaller $\Omega$ baryon size of $R_{\Omega}=0.9 \mathrm{fm}$ is then needed to keep $d N / d y$ of $\Omega$ baryons at midrapidity unchanged when the strange quark mass is increased to $500 \mathrm{MeV}$.

We also find that the larger strange quark mass of $500 \mathrm{MeV}$ leads to a slightly stiffer transverse-momentum spectra for $\phi$ mesons and $\Omega$ baryons. For $R_{\phi}=0.65(0.47) \mathrm{fm}$, the mean transverse momentum $\left\langle p_{T}\right\rangle$ for the $\phi$ meson changes from about $0.72(0.73)$ and $0.68(0.70) \mathrm{GeV} / c$ for $b=0 \mathrm{fm}$ and $b=$ $8 \mathrm{fm}$, respectively, to about $0.74(0.76)$ and $0.69(0.72) \mathrm{GeV} / c$ for $b=0 \mathrm{fm}$ and $b=8 \mathrm{fm}$, respectively, when the strange quark mass changes from 199 to $500 \mathrm{MeV}$. For $\Omega$ baryons, the value of $\left\langle p_{T}\right\rangle$ with $R_{\Omega}=1.2 \mathrm{fm}$ changes from about 0.93 and $0.83 \mathrm{GeV} / c$ for $b=0 \mathrm{fm}$ and $b=8 \mathrm{fm}$, respectively, to about 0.96 and $0.85 \mathrm{GeV} / c$ for $b=0 \mathrm{fm}$ and $b=8 \mathrm{fm}$, respectively, when the strange quark mass changes from 199 to $500 \mathrm{MeV}$.

With the new parameters $m_{s}=500 \mathrm{MeV}, R_{\phi}=0.5$ or $0.35 \mathrm{fm}$, and $R_{\Omega}=0.9 \mathrm{fm}$ fixed from measured yields of $\phi$ mesons and $\Omega$ baryons, the transverse-momentum spectra of midrapidity $\phi$ mesons and $\Omega$ baryons and their ratio in the same collision are shown in Fig. 16. As in Fig. 8, corresponding experimental data are also included in the left panels of Fig. 16. For $R_{\phi}=0.5(0.35) \mathrm{fm}$ and $m_{s}=500 \mathrm{MeV}$, the mean $\phi$ meson transverse-momentum calculated from the $\phi$ meson transverse-momentum spectrum is about $0.76(0.77)$ and 0.72 (0.74) $\mathrm{GeV} / c$ for $b=0 \mathrm{fm}$ and $b=8 \mathrm{fm}$, respectively, which are still somewhat smaller than the experimental value of about $0.85-1.1 \mathrm{GeV} / c[38,39]$. For $\Omega$ baryons with $R_{\Omega}=0.9 \mathrm{fm}$, their mean transverse momentum is about 1.05 and $0.94 \mathrm{GeV} / c$ for $b=0 \mathrm{fm}$ and $b=8 \mathrm{fm}$, respectively.

The $\Omega / \phi$ ratio obtained from these new parameters is shown in the lower panels of Fig. 16. Compared with the case of $m_{s}=199 \mathrm{MeV}$, the $\Omega / \phi$ ratio increases even more from low $p_{T}$ to intermediate $p_{T}$ of about $2.5 \mathrm{GeV} / c$, leading to a larger enhancement factor of about 14 and 9 for $b=0 \mathrm{fm}$ and $b=8 \mathrm{fm}$, respectively. The $\bar{\Omega}^{+} / \phi$ at $p_{T} \approx 2.5 \mathrm{GeV} / c$ is now about 0.30 and 0.22 for $b=0 \mathrm{fm}$ and $b=8 \mathrm{fm}$, respectively, which are still significantly smaller than that



FIG. 16. (Color online) Same as Fig. 8 but with $m_{s}=500 \mathrm{MeV}$ and $R_{\phi}=0.5$ or $0.35 \mathrm{fm}$ and $R_{\Omega}=0.9 \mathrm{fm}$.

of antiprotons to pions observed in experiments but remain comparable to the measured $\Omega / \phi$ ratio for the larger $\phi$ meson size of $R_{\phi}=0.5 \mathrm{fm}$.

\section{B. Anisotropic flows of $\phi$ and $\Omega$}

Using the fitted hadron size parameters for $m_{s}=500 \mathrm{MeV}$ (i.e., $R_{\phi}=0.5$ or $0.35 \mathrm{fm}$ and $R_{\Omega}=0.9 \mathrm{fm}$ ), we show in the left panel of Fig. 17 the $p_{T}$ dependence of the anisotropic flows $v_{2}$ (solid squares for $R_{\phi}=0.5 \mathrm{fm}$ and triangles for $R_{\phi}=0.35 \mathrm{fm}$ ) and $v_{4}$ (open squares for $R_{\phi}=0.5 \mathrm{fm}$ and triangles for $R_{\phi}=0.35 \mathrm{fm}$ ) of midrapidity $\phi$ mesons produced in $\mathrm{Au}+\mathrm{Au}$ collisions at $\sqrt{s_{N N}}=200 \mathrm{GeV}$ and $b=8 \mathrm{fm}$. Also shown in the left panel of Fig. 17 are $1.0 v_{2}^{2}$ (solid line for $R_{\phi}=0.5 \mathrm{fm}$ ) and $1.1 v_{2}^{2}$ (dashed line for $R_{\phi}=0.35 \mathrm{fm}$ ). Similar results for $\Omega$ baryons are shown in the right panel of Fig. 17. Compared to those shown in Fig. 10 with a strange

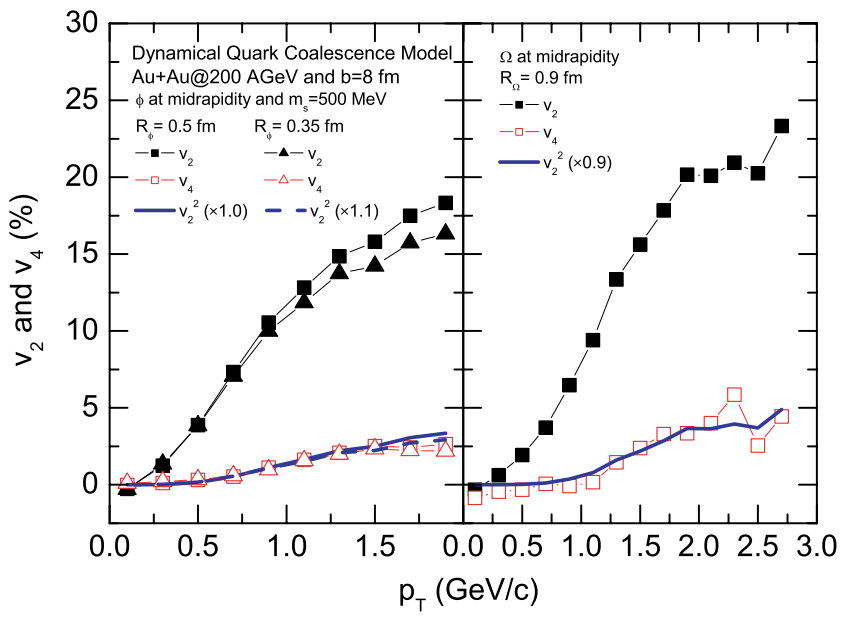

FIG. 17. (Color online) Same as Fig. 10 but with $m_{s}=500 \mathrm{MeV}$ and $R_{\phi}=0.5$ or $0.35 \mathrm{fm}$ and $R_{\Omega}=0.9 \mathrm{fm}$. 


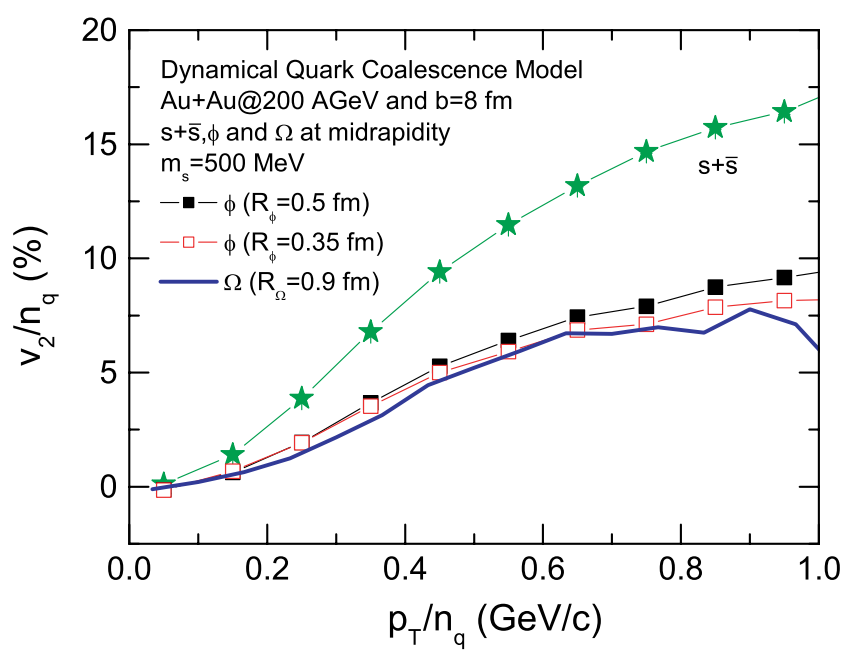

FIG. 18. (Color online) Same as Fig. 11 but with $m_{s}=500 \mathrm{MeV}$ and $R_{\phi}=0.5$ and $0.35 \mathrm{fm}$ and $R_{\Omega}=0.9 \mathrm{fm}$.

quark mass of $199 \mathrm{MeV}$, the hadron elliptic flow is seen to depend only weakly on the strange quark mass, with the larger strange quark mass of $m_{s}=500 \mathrm{MeV}$ giving a slightly smaller $v_{2}$ at lower $p_{T}$ while a slightly larger $v_{2}$ at higher $p_{T}$. Also, the anisotropic flows of $\phi$ mesons and $\Omega$ baryons from the dynamical quark coalescence model with the larger strange quark mass of $m_{s}=500 \mathrm{MeV}$ still satisfy the scaling relation $v_{4}\left(p_{T}\right) \sim v_{2}^{2}\left(p_{T}\right)$ with scaling coefficients similar to those for the strange quark mass of $199 \mathrm{MeV}$.

The $p_{T} / n_{q}$ dependence of $v_{2}$ per valence quarks or antiquarks for midrapidity $\phi$ mesons and $\Omega$ baryons produced in $\mathrm{Au}+\mathrm{Au}$ collisions at $\sqrt{s_{N N}}=200 \mathrm{GeV}$ and $b=8 \mathrm{fm}$, obtained with the large strange quark mass of $500 \mathrm{MeV}, R_{\phi}=$ 0.5 or $0.35 \mathrm{fm}$, and $R_{\Omega}=0.9 \mathrm{fm}$, is shown in Fig. 18 together with the elliptic flow of midrapidity strange and antistrange quarks $(s+\bar{s})$ at freeze-out. It is seen that the $v_{2}$ of midrapidity $\phi$ mesons and $\Omega$ baryons still satisfies approximately the valence number scaling in the dynamical quark coalescence model. However, the valence quark number scaled $v_{2}$ remains significantly smaller than the $v_{2}$ of coalescing strange and antistrange quarks.

\section{SUMMARY}

Based on the parton phase-space information obtained from a multiphase transport model within the string-melting scenario, we have studied the production of $\phi$ mesons and $\Omega$ baryons and their anisotropic flows in $\mathrm{Au}+\mathrm{Au}$ collisions at RHIC using a dynamical quark coalescence model, which requires information on the radii of the $\phi$ meson and the $\Omega$ baryon. Fixing their radii by fitting measured yields of $\phi$ mesons and $\Omega$ baryons at midrapidity in central $\mathrm{Au}+\mathrm{Au}$ collisions at $\sqrt{s_{N N}}=200 \mathrm{GeV}$, we have evaluated their transverse-momentum spectra in the same collision at impact parameter $b=0$ and $8 \mathrm{fm}$ and also their anisotropic flows in the same collision at impact parameter $b=8 \mathrm{fm}$. Comparing with its value at low transverse momenta, we have found that the ratio of the yield of $\Omega$ baryons to that of $\phi$ mesons is enhanced significantly at intermediate transverse momenta as observed in experiments.
We have further found that the elliptic flows of $\phi$ mesons and $\Omega$ baryons follow approximately the valence quark number scaling. The valence quark number scaled elliptic flows of $\phi$ mesons and $\Omega$ baryons deviate, however, strongly from the underlying $v_{2}$ of strange and antistrange quarks. Moreover, we have also studied the fourth-order anisotropic flow $v_{4}$ and found that the scaling relation of $v_{4}\left(p_{T}\right) \sim v_{2}^{2}\left(p_{T}\right)$ observed experimentally for charged hadrons is satisfied by $\phi$ mesons and $\Omega$ baryons as well. It will be very interesting to compare these predictions with experimental data that are being analyzed.

In addition, we have studied the dependence of these results on the radii of the $\phi$ meson and the $\Omega$ baryon as well as the strange quark mass. Both the yields and anisotropic flows of $\phi$ mesons and $\Omega$ baryons are found to be sensitive to their radii. For sufficient large radii, the valence quark number scaled elliptic flows of $\phi$ mesons and $\Omega$ baryons approach the $v_{2}$ of strange and antistrange quarks as in the naive momentum-space coalescence model. Also the scaling coefficient $v_{4}\left(p_{T}\right) / v_{2}^{2}\left(p_{T}\right)$ is sensitive to hadron size. Although the strange quark mass was found to affect significantly the yields of $\phi$ mesons and $\Omega$ baryons, it does not change much their anisotropic flows. Our results thus suggest that in using the quark coalescence model to extract the parton dynamics in relativistic heavy-ion collisions from hadron observables, it is important to take into account the quark structure of hadrons.

Although results from the present study reproduce reasonably the observed transverse-momentum dependence of the $\Omega / \phi$ ratio, the transverse-momentum spectra of $\phi$ mesons and $\Omega$ baryons are too soft compared with measured ones. This has been attributed to the small current quark masses used in the AMPT model. Recent studies have shown that the equation of state of the QGP from lattice QCD calculations can only be reproduced by partons with large masses $[67,68]$. It is thus of great interest to improve the AMPT model by using massive partons and to study how the resulting parton dynamics affects the results obtained in the present study. Although we have shown in the present study that the anisotropic flows of $\phi$ mesons and $\Omega$ baryons are not sensitive to the strange quark mass used in the dynamical coalescence model if the same strange quark distribution is used, using massive partons in the AMPT model not only is expected to affect the transversemomentum spectra of partons but also may influence their anisotropic flow and thus that of produced hadrons. Also, it is important to include in future studies the contribution from high-momentum jets [69] as they are expected to affect the production of $\phi$ mesons and $\Omega$ baryons as well as their anisotropic flows at intermediate and high transverse momenta.

\section{ACKNOWLEDGMENTS}

We thank Professor Zong-Ye Zhang for helpful communications on the radius of the $\Omega$ baryon in the quark model. This work was supported in part by the National Natural Science Foundation of China under Grant Nos. 10105008 and 10575071 and by MOE of China under project NCET-05-0392 (LWC) as well as by the US National Science Foundation under Grant No. PHY-0457265 and the Welch Foundation under Grant No. A-1358 (CMK). 
[1] V. Greco, C. M. Ko, and P. Lévai, Phys. Rev. Lett. 90, 202302 (2003); Phys. Rev. C 68, 034904 (2003).

[2] R. C. Hwa and C. B. Yang, Phys. Rev. C 67, 034902 (2003); 67, 064902 (2003).

[3] R. J. Fries, B. Müller, C. Nonaka, and S. A. Bass, Phys. Rev. Lett. 90, 202303 (2003); Phys. Rev. C 68, 044902 (2003).

[4] D. Molnar and S. A. Voloshin, Phys. Rev. Lett. 91, 092301 (2003).

[5] D. Molnar, J. Phys. G 30, S1239 (2004); 31, S421 (2005).

[6] J. Rafelski and B. Müller, Phys. Rev. Lett. 48, 1066 (1982); Phys. Lett. B101, 111 (1982).

[7] P. Koch, B. Müller, and J. Rafelski, Phys. Rep. 142, 167 (1986), and references therein.

[8] J. H. Chen et al., nucl-th/0504055.

[9] J. Adams et al. (STAR Collaboration), Phys. Rev. Lett. 95, 122301 (2005).

[10] J. Y. Ollitrault, Phys. Rev. D 46, 229 (1992).

[11] H. Sorge, Phys. Lett. B402, 251 (1997); Phys. Rev. Lett. 78, 2309 (1997); 82, 2048 (1999).

[12] P. Danielewicz, R. A. Lacey, P. B. Gossiaux, C. Pinkenburg, P. Chung, J. M. Alexander, and R. L. McGrath, Phys. Rev. Lett. 81, 2438 (1998).

[13] Y. M. Zheng, C. M. Ko, B. A. Li, and B. Zhang, Phys. Rev. Lett. 83, 2534 (1999).

[14] P. F. Kolb, P. Huovinen, U. Heinz, and H. Heiselberg, Phys. Lett. B500, 232 (2001).

[15] P. Huovinen, P. F. Kolb, U. Heinz, P. V. Ruuskanen, and S. Voloshin, Phys. Lett. B503, 58 (2001).

[16] B. Zhang, M. Gyulassy, and C. M. Ko, Phys. Lett. B455, 45 (1999).

[17] D. Molnar and M. Gyulassy, Nucl. Phys. A698, 379 (2002).

[18] Z. W. Lin and C. M. Ko, Phys. Rev. C 65, 034904 (2002).

[19] M. Gyulassy, I. Vitev, and X. N. Wang, Phys. Rev. Lett. 86, 2537 (2001).

[20] S. A. Voloshin, Nucl. Phys. A715, 379c (2003).

[21] D. Teaney, J. Lauret, and E. V. Shuryak, Phys. Rev. Lett. 86, 4783 (2001).

[22] P. F. Kolb, J. Sollfrank, and U. Heinz, Phys. Lett. B459, 667 (1999).

[23] D. Teaney and E. V. Shuryak, Phys. Rev. Lett. 83, 4951 (1999).

[24] P. F. Kolb, J. Sollfrank, and U. Heinz, Phys. Rev. C 62, 054909 (2000).

[25] P. F. Kolb, Phys. Rev. C 68, 031902(R) (2003).

[26] J. Adams et al. (STAR Collaboration), Phys. Rev. Lett. 92, 062301 (2004).

[27] J. Adams et al. (STAR Collaboration), Phys. Rev. C 72, 014904 (2005).

[28] L. W. Chen, C. M. Ko, and Z. W. Lin, Phys. Rev. C 69, 031901(R) (2004).

[29] P. F. Kolb, L. W. Chen, V. Greco, and C. M. Ko, Phys. Rev. C 69, 051901(R) (2004).

[30] A. Shor, Phys. Rev. Lett. 54, 1122 (1985).

[31] C. P. Singh, Phys. Rev. Lett. 56, 1750 (1986).

[32] H. van Hecke, H. Sorge, and N. Xu, Phys. Rev. Lett. 81, 5764 (1998).

[33] S. A. Bass, A. Dumitru, M. Bleicher, L. Bravina, E. Zabrodin, H. Stöcker, and W. Greiner, Phys. Rev. C 60, 021902(R) (1999); A. Dumitru, S. A. Bass, M. Bleicher, H. Stöcker, W. Greiner, Phys. Lett. B460, 411 (1999); S. A. Bass and A. Dumitru, Phys. Rev. C 61, 064909 (2000).

[34] Y. Cheng, F. Liu, Z. Liu, K. Schweda, and N. Xu, Phys. Rev. C 68, 034910 (2003).
[35] S. F. Biagi et al., Nucl. Phys. B186, 1 (1981).

[36] R. A. Muller, Phys. Lett. B38, 123 (1972).

[37] J. Adams et al. (STAR Collaboration), Phys. Rev. Lett. 92, 182301 (2004)

[38] A. Adams et al. (STAR Collaboration), Phys. Lett. B612, 181 (2005).

[39] S. S. Adler et al. (PHENIX Collaboration), Phys. Rev. C 72, 014903 (2002); D. Mukhopadhyay for the PHENIX Collaboration, J. Phys. G 31, S187 (2005).

[40] C. Suire et al. (STAR Collaboration), Nucl. Phys. A715, 470c (2003).

[41] Z. W. Lin, C. M. Ko, B. A. Li, B. Zhang, and S. Pal, Phys. Rev. C 72, 064901 (2005).

[42] X. N. Wang and M. Gyulassy, Phys. Rev. D 44, 3501 (1991).

[43] B. Zhang, Comput. Phys. Commun. 109, 193 (1998).

[44] T. Sjostrand, Comput. Phys. Commun. 82, 74 (1994).

[45] B. A. Li and C. M. Ko, Phys. Rev. C 52, 2037 (1995); B. A. Li, A. T. Sustich, B. Zhang, and C. M. Ko, Int. J. Phys. E 10, 267 (2001).

[46] B. Zhang, C. M. Ko, B. A. Li, Z. Lin, and B. H. Sa, Phys. Rev. C 62, 054905 (2000); B. Zhang, C. M. Ko, B. A. Li, Z. W. Lin, and S. Pal, ibid. 65, 054909 (2002).

[47] L. W. Chen and C. M. Ko, Phys. Lett. B634, 205 (2006).

[48] D. Kharzeev and M. Nardi, Phys. Lett. B507, 121 (2001).

[49] Z. W. Lin, C. M. Ko, and S. Pal, Phys. Rev. Lett. 89, 152301 (2002).

[50] K. H. Ackermann et al. (STAR Collaboration), Phys. Rev. Lett. 86, 402 (2001).

[51] C. Adler et al. (STAR Collaboration), Phys. Rev. Lett. 87, 082301 (2001)

[52] F. Karsch, Nucl. Phys. A698, 199c (2002).

[53] Z. Fodor, Nucl. Phys. A715, 319c (2003), and references therein; Z. Fodor and S. D. Katz, Phys. Lett. B534, 87 (2002).

[54] J. Barrette et al. (E877 Collaboration), Phys. Rev. Lett. 73, 2532 (1994).

[55] H. Appelshauser et al. (NA49 Collaboration), Phys. Rev. Lett. 80, 4136 (1998).

[56] R. Mattiello, A. Jahns, H. Sorge, H. Stocker, and W. Greiner, Phys. Rev. Lett. 74, 2180 (1995); R. Mattiello, H. Sorge, H. Stocker, and W. Greiner, Phys. Rev. C 55, 1443 (1997).

[57] L. W. Chen, C. M. Ko, and B. A. Li, Phys. Rev. C 68, 017601 (2003); Nucl. Phys. A729, 809 (2003).

[58] L. W. Chen, V. Greco, C. M. Ko, S. H. Lee, and W. Liu, Phys. Lett. B601, 34 (2004).

[59] A. T. M Aerts and C. B. Dover, Phys. Rev. D 28, 450 (1983).

[60] R. Scheibl and U. Heinz, Phys. Rev. C 59, 1585 (1999).

[61] L. W. Chen, V. Greco, C. M. Ko, and P. F. Kolb, Phys. Lett. B605, 95 (2005)

[62] S. S. Adler et al. (PHEHIX Collaboration), Phys. Rev. C 69, 034909 (2004)

[63] V. Greco and C. M. Ko, Phys. Rev. C 70, 024901 (2004).

[64] V. Greco and C. M. Ko, arXiv:nucl-th/0505061.

[65] S. Pratt and S. Pal, Phys. Rev. C 71, 014905 (2005); Nucl. Phys. A749, 268c (2005).

[66] B. Muller, R. J. Fries, and S. A. Bass, Phys. Lett. B618, 77 (2005).

[67] P. Levai and U. Heinz, Phys. Rev. C 57, 1879 (1998).

[68] A. Peshier and W. Cassing, Phys. Rev. Lett. 94, 172301 (2005).

[69] V. Greco, C. M. Ko, and I. Vitev, Phys. Rev. C 71, 041901(R) (2005). 